\title{
Multiple Solutions for a Fractional Laplacian System Involving Critical Sobolev-Hardy Exponents and Homogeneous Term
}

\author{
Jinguo Zhang ${ }^{a}$ and Tsing-San $\mathrm{Hsu}^{b}$ \\ ${ }^{a}$ School of Mathematics, Jiangxi Normal University \\ Nanchang 330022, China \\ ${ }^{b}$ Center for General Education, Chang Gung University \\ Tao-Yuan, Taiwan \\ E-mail(corresp.): jgzhang@jxnu.edu.cn \\ E-mail: tshsu@mail.cgu.edu.tw
}

Received January 16, 2019; revised October 9, 2019; accepted October 11, 2019

\begin{abstract}
In this paper, we deal with a class of fractional Laplacian system with critical Sobolev-Hardy exponents and sign-changing weight functions in a bounded domain. By exploiting the Nehari manifold and variational methods, some new existence and multiplicity results are obtain.
\end{abstract}

Keywords: fractional Laplacian system, Nehari manifold, critical Sobolev-Hardy exponent, homogeneous term.

AMS Subject Classification: 35J20; 35J60.

\section{Introduction}

In this paper, we mainly study the following system of fractional elliptic equations:

$$
\begin{cases}(-\Delta)^{s} u-\gamma \frac{u}{|x|^{2 s}}=\lambda f(x) \frac{|u|^{q-2} u}{|x|^{\alpha}}+\frac{1}{2_{s}^{*}(\beta)} \frac{F_{u}(u, v)}{|x|^{\beta}}, & \text { in } \Omega, \\ (-\Delta)^{s} v-\gamma \frac{v}{|x|^{2 s}}=\mu g(x) \frac{|v|^{q-2} v}{|x|^{\alpha}}+\frac{1}{2_{s}^{*}(\beta)} \frac{F_{v}(u, v)}{|x|^{\beta}}, & \text { in } \Omega, \\ u=v=0, & \text { in } \mathbb{R}^{N} \backslash \Omega,\end{cases}
$$

where $s \in(0,1), \Omega \subset \mathbb{R}^{N}(N>2 s)$ is a smooth bounded domain with $0 \in \Omega$, $2_{s}^{*}(\beta):=2(N-\beta) /(N-2 s)$ is the fractional Sobolev-Hardy critical exponent, 
and the parameters in (1.1) satisfy the following assumptions:

$$
N>2 s, 0<s<1,0 \leq \alpha, \beta<2 s, \lambda, \mu>0,0 \leq \gamma<\gamma_{H}, 1 \leq q<2 .
$$

The operator $(-\Delta)^{s}$ is the fractional Laplacian which is defined by

$$
\begin{aligned}
(-\Delta)^{s} u(x) & =\mathrm{P} . \mathrm{V} \cdot \int_{\mathbb{R}^{N}} \frac{u(x)-u(y)}{|x-y|^{N+2 s}} d y \\
& =\lim _{\varepsilon \rightarrow 0^{+}} \int_{\mathbb{R}^{N} \backslash B_{\varepsilon}(x)} \frac{u(x)-u(y)}{|x-y|^{N+2 s}} d y, \quad \forall x \in \mathbb{R}^{N} .
\end{aligned}
$$

Notice that the typical feature of the fractional Laplacian operator is nonlocality, that is, the value $(-\Delta)^{s} u(x)$ at any point $x \in \Omega$ depends not only on the value of $u$ on the $\Omega$, but also on the value of $u$ on the whole $\mathbb{R}^{N}$, which makes some discussions and calculations difficult. Moreover, the Dirichlet condition in (1.1) is given in $\mathbb{R}^{N} \backslash \Omega$ and not simply on $\partial \Omega$, which consistently with the nonlocal character of the operator $(-\Delta)^{s}$, see $[1,3,16,17,19,21]$ and the references therein for further details on the fractional Laplacian.

The starting point on the study of the system (1.1) is its scalar version:

$$
\begin{cases}(-\Delta)^{s} u-\gamma \frac{u}{|x|^{2 s}}=\lambda f(x) \frac{|u|^{q-2} u}{|x|^{\alpha}}+\frac{|u|^{2_{s}^{*}(\beta)-2} u}{|x|^{\beta}}, & \text { in } \Omega, \\ u=0, & \text { in } \mathbb{R}^{N} \backslash \Omega .\end{cases}
$$

Concerning the nonlocal problems with critical Sobolev-Hardy exponents, there has been little research up to now, see $[18,20]$ and the references therein. In particular, Zhang and Hsu [20] concerned the following fractional elliptic system

$$
\begin{cases}(-\Delta)^{s} u-\gamma \frac{u}{|x|^{2 s}}=\lambda \frac{|u|^{q-2} u}{|x|^{\alpha}}+\frac{2 \eta}{\eta+\theta} \frac{|u|^{\eta-2} u|v|^{\theta}}{|x|^{\beta}}, & \text { in } \Omega, \\ (-\Delta)^{s} v-\gamma \frac{v}{|x|^{2 s}}=\mu \frac{|v|^{q-2} v}{|x|^{\alpha}}+\frac{2 \theta}{\eta+\theta} \frac{|u|^{\eta}|v|^{\theta-2} v}{|x|^{\beta}}, & \text { in } \Omega, \\ u=v=0, & \text { in } \mathbb{R}^{N} \backslash \Omega,\end{cases}
$$

where $1 \leq q<2, \eta, \theta>1$ satisfying $\eta+\theta=2_{s}^{*}(\beta)$. Using the variational method and Nehari manifold method, they found that the problem (1.3) has at least two positive solutions if the parameters $\lambda, \mu>0$ satisfied a certain condition. Problems (1.2) and (1.3) aroused the interesting results due to the lack of compactness for involving the critical exponent; hence, the associated energy functionals do not satisfy the Palais-Smale condition in general. Moreover, the explicit formula of the ground states of limiting problem (2.9) is not clear, the standard variational argument is not applicable directly, which is the difficulty for the fractional Laplacian problem with Hardy potential and critical growth.

Motivated by [20], in this paper we focus on the general case $f, g$ possibly change sign in $\Omega$ and $F$ positively $2_{s}^{*}(\beta)$-homogeneous, we shall complement the results of $[18,20]$ and extend the results of $[11,12,13,14]$ to the fractional Laplacian operator. Our main tool is the Nehari manifold methods which is 
similar to the fibering method of Drabek and Pohozaev [5]. We show that the system (1.1) has at least two positive solutions when the parameters $\lambda, \mu$ and weight functions $f, g$ satisfied some certain conditions. It should be mentioned that in $[8,9,10,15,22]$, some problems involving fractional Laplacian operator were investigated by the Nehari manifold and fibering method.

We look for solutions of (1.1) in the Sobolev space

$$
X_{0}^{s}(\Omega)=\left\{u \in H^{s}\left(\mathbb{R}^{N}\right): u=0 \text { a.e. in } \mathbb{R}^{N} \backslash \Omega\right\}
$$

with the norm

$$
\|u\|_{X_{0}^{s}(\Omega)}:=\left(\int_{\mathbb{R}^{N}} \int_{\mathbb{R}^{N}} \frac{|u(x)-u(y)|^{2}}{|x-y|^{N+2 s}} d x d y\right)^{\frac{1}{2}} .
$$

From (2.2), we employ the following equivalent norm in $X_{0}^{s}(\Omega)$ :

$$
\|u\|_{\gamma}=\left(\int_{\mathbb{R}^{N}} \int_{\mathbb{R}^{N}} \frac{|u(x)-u(y)|^{2}}{|x-y|^{N+2 s}} d x d y-\gamma \int_{\Omega} \frac{|u|^{2}}{|x|^{2 s}} d x\right)^{\frac{1}{2}} .
$$

Denote by $W$ the product space $W=X_{0}^{s}(\Omega) \times X_{0}^{s}(\Omega)$ endowed with the norm $\|(u, v)\|_{W}^{2}=\|u\|_{\gamma}^{2}+\|v\|_{\gamma}^{2}$. The corresponding energy functional of problem (1.1) is defined on $W$ by

$$
I_{\lambda, \mu}(u, v)=\frac{1}{2}\|(u, v)\|_{W}^{2}-\frac{1}{2_{s}^{*}(\beta)} \int_{\Omega} \frac{F(u, v)}{|x|^{\beta}} d x-\frac{1}{q} Q_{\lambda, \mu}(u, v),
$$

where

$$
Q_{\lambda, \mu}(u, v)=\lambda \int_{\Omega} \frac{f(x)|u|^{q}}{|x|^{\alpha}} d x+\mu \int_{\Omega} \frac{g(x)|v|^{q}}{|x|^{\alpha}} d x .
$$

By standard arguments we can verify $I_{\lambda, \mu} \in C^{1}(W, \mathbb{R})$. It is well-known that the weak solutions of (1.1) are the critical points of functional $I_{\lambda, \mu}$.

For all $\gamma<\gamma_{H}$, by $\left(\mathcal{H}_{0}\right)$, the following best Sobolev-type constants are well defined and crucial for the study of (1.1):

$$
\begin{aligned}
& \Lambda(s, \beta):=\inf _{u \in X_{0}^{s}(\Omega) \backslash\{0\}} \frac{\int_{\mathbb{R}^{N}} \int_{\mathbb{R}^{N}} \frac{|u(x)-u(y)|^{2}}{|x-y|^{N+2 s}} d x d y-\gamma \int_{\Omega} \frac{|u|^{2}}{|x|^{2 s}} d x}{\left(\int_{\Omega} \frac{|u|_{s}^{2_{s}^{*}(\beta)}}{|x|^{\beta}} d x\right)^{\frac{2}{2_{s}^{*}(\beta)}}}, \\
& S_{F}(s, \beta):=\inf _{u \in W \backslash\{(0,0)\}}\|(u, v)\|_{W}^{2} /\left(\int_{\Omega} \frac{F(u, v)}{|x|^{\beta}} d x\right)^{\frac{2}{2_{s}^{*}(\beta)}} .
\end{aligned}
$$

In order to given the relation between $\Lambda(s, \beta)$ and $S_{F}(s, \beta)$, the following assumptions on $F$ are needed in this paper:

$\left(F_{0}\right) F \in C^{1}\left(\mathbb{R}^{2}, \mathbb{R}^{+}\right)$and $F(t u, t v)=t^{2_{s}^{*}(\beta)} F(u, v), \forall t \geq 0,(u, v) \in \mathbb{R}^{2} ;$

$\left(F_{1}\right) F(u, 0)=F(0, v)=F_{u}(u, 0)=F_{v}(0, v)=0, \forall u, v \in \mathbb{R} ;$

$\left(F_{2}\right) F_{u}(u, v), F_{v}(u, v)$ are strictly increasing functions for all $(u, v) \in \mathbb{R}^{2}$.

Then, we have the following result. 
Theorem 1. Suppose $\left(\mathcal{H}_{0}\right)$ and $\left(F_{0}\right)-\left(F_{2}\right)$ hold. Then, $S_{F}(s, \beta)=M_{F}^{-1} \Lambda(s, \beta)$ and $S_{F}(s, \beta)$ has the minimizers $\left(\theta_{1} U_{\varepsilon}(x), \theta_{2} U_{\varepsilon}(x)\right)$, where $U_{\varepsilon}(x)$ are defined as in (2.8), $\theta_{1}, \theta_{2}$ are constants given in (2.4), and $M_{F}$ is defined by

$$
M_{F}=\max \left\{F(|u|,|v|)^{\frac{2}{2_{s}^{*}(\beta)}}:(u, v) \in \mathbb{R}^{2} \text { and }|u|^{2}+|v|^{2}=1\right\} .
$$

The other main result of this paper is the following existence and multiple results. To the best of our knowledge, the results are new for the critical fractional Laplacian problem with Hardy potential and homogeneous term.

Set

$$
\Lambda_{0}=\left(\frac{2-q}{2_{s}^{*}(\beta)-q}\right)^{\frac{2}{2 *(\beta)-2}} S_{F}(s, \beta)^{\frac{2_{s}^{*}(\beta)}{2 s(\beta)-2}}\left(\frac{2_{s}^{*}(\beta)-2}{2_{s}^{*}(\beta)-q}\right)^{\frac{2}{2-q}} \Lambda(s, \alpha)^{\frac{q}{2-q}} .
$$

We assume that $f, g: \Omega \rightarrow \mathbb{R}$ satisfy

$\left(\mathcal{H}_{1}\right) f, g \in L^{p}\left(\Omega,|x|^{-\alpha} d x\right), f^{ \pm}=\max \{ \pm f, 0\} \neq 0$ and $g^{ \pm}=\max \{ \pm g, 0\} \neq 0$, where $p:=2_{s}^{*}(\alpha) /\left(2_{s}^{*}(\alpha)-q\right)$.

$\left(\mathcal{H}_{2}\right)$ For some $a_{0}, r_{0}>0$ such that $f(x), g(x) \geq a_{0}$ for $x \in B_{r_{0}}(0) \subset \Omega$.

Theorem 2. Suppose $\left(\mathcal{H}_{0}\right),\left(\mathcal{H}_{1}\right)-\left(\mathcal{H}_{2}\right)$ and $\left(F_{0}\right)-\left(F_{2}\right)$ hold. Then,

(i) If $\lambda, \mu>0$ satisfy

$$
0<\left(\lambda\|f\|_{L^{p}\left(\Omega,|x|^{-\alpha}\right)}\right)^{\frac{2}{2-q}}+\left(\mu\|f\|_{L^{p}\left(\Omega,|x|^{-\alpha}\right)}\right)^{\frac{2}{2-q}}<A_{0},
$$

then system (1.1) has at least one positive solution in $W$.

(ii) There exists $0<A^{*}<A_{0}$ such that for $\lambda, \mu>0$ and

$$
0<\left(\lambda\|f\|_{L^{p}\left(\Omega,|x|^{-\alpha}\right)}\right)^{\frac{2}{2-q}}+\left(\mu\|f\|_{L^{p}\left(\Omega,|x|^{-\alpha}\right)}\right)^{\frac{2}{2-q}}<A^{*},
$$

then problem (1.1) has at least two positive solutions in $W$.

Remark 1. There are many homogeneous functions of class $C^{1}$, for example: $F(t)=\left(\sum_{i=1}^{k}\left|t_{i}\right|^{p}\right)^{\frac{2_{s}^{*}(\beta)}{p}}$ with $p \geq 1$. If we taking $F(\mathbf{u})=\frac{2}{\eta+\theta}\left|u_{1}\right|^{\eta}\left|u_{2}\right|^{\theta}$ with $\eta$, $\theta>1, \eta+\theta=2_{s}^{*}(\beta)$, then Theorems 1.1 and 1.2 in [20] are the special case of our Theorems 1 and 2 .

This paper is organized as follows. In Section 2, we introduce the variational setting of the problem and present some norm estimates about the ground states of limiting problem. In Section 3, we investigate the Palais-Smale condition for the energy functional and given the proof of Theorem 1. Some properties about the fibering maps and Nehari manifold are established in Section 4, and Theorem 2 is proved in Sections 5.

Throughout this paper, we will denote by $L^{q}\left(\Omega,|x|^{\alpha} d x\right)$ the usual weighted $L^{q}(\Omega)$ space with the weight $|x|^{\alpha}$ which norm give by $\|\cdot\|_{L^{q}\left(\Omega,|x|^{\alpha}\right)} ; O\left(\varepsilon^{t}\right)$ denotes $\frac{\left|O\left(\varepsilon^{t}\right)\right|}{\varepsilon^{t}} \leq C$ and $o\left(\varepsilon^{t}\right)$ means $\frac{\left|o\left(\varepsilon^{t}\right)\right|}{\varepsilon^{t}} \rightarrow 0$ as $\varepsilon \rightarrow 0$ for $t \geq 0 ; o_{n}(1)$ means $o_{n}(1) \rightarrow 0$ as $n \rightarrow \infty$; The dual space of $W$ will be denoted by $W^{-1} ; C$, $C_{i}$ will denote various positive constants which may vary from line to line. 


\section{Preliminaries}

First, we give some useful results of fractional Sobolev-Hardy inequality. Assume that $0 \leq t \leq 2 s$. Then, there exists positive constant $C(s, t, N)$ depending on $s, t$ and $N$, such that

$$
C(s, t, N)\left(\int_{\mathbb{R}^{N}} \frac{|u|^{2_{s}^{*}(t)}}{|x|^{t}} d x\right)^{\frac{2}{2_{s}^{*}(t)}} \leq \int_{\mathbb{R}^{N}} \int_{\mathbb{R}^{N}} \frac{|u(x)-u(y)|^{2}}{|x-y|^{N+2 s}} d x d y,
$$

where $2_{s}^{*}(t)=\frac{2(N-t)}{N-2 s}$. If we set $t=2 s$ in $(2.1)$, we have

$$
\gamma_{H} \int_{\Omega} \frac{|u|^{2}}{|x|^{2 s}} d x \leq \int_{\mathbb{R}^{N}} \int_{\mathbb{R}^{N}} \frac{|u(x)-u(y)|^{2}}{|x-y|^{N+2 s}} d x d y, \quad \forall u \in X_{0}^{s}(\Omega),
$$

where $\gamma_{H}:=2^{2 s} \frac{\Gamma^{2}\left(\frac{N+2 s}{4}\right)}{\Gamma^{2}\left(\frac{N-2 s}{4}\right)}$ is the best fractional Hardy constant.

The following properties about homogeneous function are important and well known:

Lemma 1. Let $\sigma \geq 1$ and $H$ be a differentiable $\sigma$-homogeneous function, then

(i) for all $(u, v) \in \mathbb{R}^{2}, u H_{u}(u, v)+v H_{v}(u, v)=\sigma H(u, v)$;

(ii) there exists $M_{H}>0$ such that $|H(u, v)| \leq M_{H}\left(|u|^{\sigma}+|v|^{\sigma}\right)$ for all $(u, v) \in$ $\mathbb{R}^{2}$, where

$$
M_{H}=\max \left\{H(u, v):(u, v) \in \mathbb{R}^{2},|u|^{\sigma}+|v|^{\sigma}=1\right\}
$$

(iii) the maximum $M_{H}$ is attained for some $\left(u_{0}, v_{0}\right) \in \mathbb{R}^{2}$, i.e., $\left|u_{0}\right|^{\sigma}+\left|v_{0}\right|^{\sigma}=1$ and $\left|H\left(u_{0}, v_{0}\right)\right|=M_{H}$;

(iv) $H_{u}(u, v), H_{v}(u, v)$ are $(\sigma-1)$ homogenous.

By $\left(F_{0}\right)$ and Lemma 1 , we have

$$
u F_{u}(u, v)+v F_{v}(u, v)=2_{s}^{*}(\beta) F(u, v), F(u, v) \leq\left(M_{F}\left(|u|^{2}+|v|^{2}\right)\right)^{\frac{2_{s}^{*}(\beta)}{2}},
$$

where $M_{F}$ is given in (1.5). Moreover, from Lemma 1 (iii), there exists $\left(\theta_{1}, \theta_{2}\right) \in$ $\mathbb{R}^{2}$ such that

$$
\theta_{1}^{2}+\theta_{2}^{2}=1 \text { and } M_{F}=F\left(\theta_{1}, \theta_{2}\right)^{\frac{2}{2 *(\beta)}} .
$$

Now, we will study $S_{F}(s, \beta)$ and prove Theorem 1 .

Proof of Theorem 1. Let $\left\{w_{n}\right\} \subset X_{0}^{s}(\Omega)$ be a minimizing sequence for $\Lambda(s, \beta)$ and $\left(\theta_{1}, \theta_{2}\right)$ be defined as in $(2.4)$. Choosing $\left(u_{n}, v_{n}\right)=\left(\theta_{1} w_{n}, \theta_{2} w_{n}\right)$ in $(1.4)$, from $\left(F_{0}\right)$ we have

$$
\frac{\left(\theta_{1}^{2}+\theta_{2}^{2}\right)\left(\int_{\mathbb{R}^{N}} \int_{\mathbb{R}^{N}} \frac{\left|w_{n}(x)-w_{n}(y)\right|^{2}}{|x-y|^{N+2 s}} d x d y-\gamma \int_{\Omega} \frac{\left|w_{n}\right|^{2}}{|x|^{2 s}} d x\right)}{F\left(\theta_{1}, \theta_{2}\right)^{\frac{2}{2_{s}^{*}(\beta)}}\left(\int_{\Omega} \frac{\left|w_{n}\right|_{s}^{2_{s}^{*}(\beta)}}{|x|^{\beta}} d x\right)^{\frac{2}{2_{s}^{*}(\beta)}}} \geq S_{F}(s, \beta) .
$$


Taking $n \rightarrow \infty$ in (2.5), we have

$$
S_{F}(s, \beta) \leq M_{F}^{-1} \Lambda(s, \beta) .
$$

On the other hand, let $\left\{\left(u_{n}, v_{n}\right)\right\} \subset W \backslash\{(0,0)\}$ be a minimizing sequence for $S_{F}(s, \beta)$, from Proposition 1 in [4], we have

$$
\begin{aligned}
\int_{\Omega} \frac{F\left(u_{n}, v_{n}\right)}{|x|^{\beta}} d x & =\int_{\Omega} F\left(\frac{u_{n}}{|x|^{\frac{\beta}{2_{s}^{*}(\beta)}}}, \frac{v_{n}}{|x|^{\frac{\beta}{2_{s}^{*}(\beta)}}}\right) d x \\
& \leq F\left(\left\|\frac{u_{n}}{|x|^{\frac{\beta}{2_{s}^{*}(\beta)}}}\right\|_{L^{2_{s}^{*}(\beta)}},\left\|\frac{v_{n}}{|x|^{\frac{\beta}{2_{s}^{*}(\beta)}}}\right\|_{L_{s}^{2_{s}^{*}(\beta)}}\right) .
\end{aligned}
$$

Set

$$
\mathcal{A}=1 /\left(\left\|\frac{u_{n}}{|x|^{\frac{\beta}{2 *(\beta)}}}\right\|_{L^{2 *(\beta)}}^{2}+\left\|\frac{v_{n}}{|x|^{\frac{\beta}{2_{s}^{*}(\beta)}}}\right\|_{L^{2 *(\beta)}}^{2}\right)^{1 / 2}
$$

We have

$$
\left\|\frac{\mathcal{A} u_{n}}{|x|^{\frac{\beta}{2_{s}^{*}(\beta)}}}\right\|_{L_{s}^{2_{s}^{*}(\beta)}}^{2}+\left\|\frac{\mathcal{A} v_{n}}{|x|^{\frac{\beta}{2_{s}^{*}(\beta)}}}\right\|_{L_{s}^{2_{s}^{*}(\beta)}}^{2}=1 .
$$

Then,

$$
\begin{aligned}
& \frac{\int_{\mathbb{R}^{N}} \int_{\mathbb{R}^{N}}\left(\frac{\left|u_{n}(x)-u_{n}(y)\right|^{2}}{|x-y|^{N+2 s}}+\frac{\left|v_{n}(x)-v_{n}(y)\right|^{2}}{|x-y|^{N+2 s}}\right) d x d y-\gamma \int_{\Omega} \frac{\left|u_{n}\right|^{2}+\left|v_{n}\right|^{2}}{|x|^{2 s}} d x}{\left(\int_{\Omega} \frac{F\left(u_{n}, v_{n}\right)}{|x|^{\beta}} d x\right)^{\frac{2}{2_{s}^{*}(\beta)}}} \\
& \geq \frac{\Lambda(s, \beta)\left(\int_{\Omega} \frac{\left|u_{n}\right|^{2_{s}^{*}(\beta)}}{|x|^{\beta}} d x\right)^{\frac{2}{2_{s}^{*}(\beta)}}+\Lambda(s, \beta)\left(\int_{\Omega} \frac{\left|v_{n}\right|_{s}^{2_{s}^{*}(\beta)}}{|x|^{\beta}} d x\right)^{\frac{2}{2_{s}^{*}(\beta)}}}{\left(F\left(\left\|\frac{u_{n}}{|x|^{\frac{\beta}{2_{s}^{*}(\beta)}}}\right\|_{L_{s}^{2_{s}^{*}(\beta)}},\left\|\frac{v_{n}}{|x|^{\frac{\beta}{2_{s}^{*}(\beta)}}}\right\|_{L_{s}^{2_{s}^{*}(\beta)}}\right)\right)^{\frac{2}{2_{s}^{*}(\beta)}}} \\
& =\Lambda(s, \beta) \frac{\left\|\frac{u_{n}}{|x|^{\frac{\beta}{2_{s}^{*}(\beta)}}}\right\|_{L^{2_{s}^{*}(\beta)}}^{2}+\left\|\frac{v_{n}}{|x|^{\frac{\beta}{2^{*}(\beta)}}}\right\|_{L^{2_{s}^{*}(\beta)}}^{2}}{\left(F\left(\left\|\frac{u_{n}}{|x|^{\frac{\beta}{2_{s}^{*}(\beta)}}}\right\|_{L^{2_{s}^{*}(\beta)}},\left\|\frac{v_{n}}{|x|^{\frac{\beta}{2_{s}^{*}(\beta)}}}\right\|_{L_{s}^{2_{s}^{*}(\beta)}}\right)\right)^{\frac{2}{2_{s}^{*}(\beta)}}} \\
& =\Lambda(s, \beta) \frac{\left\|\frac{\mathcal{A} u_{n}}{|x|^{\frac{\beta}{2_{s}^{*}(\beta)}}}\right\|_{L^{2_{s}^{*}(\beta)}}^{2}+\left\|\frac{\mathcal{A} v_{n}}{|x|^{\frac{\beta}{2 *(\beta)}}}\right\|_{L^{2_{s}^{*}(\beta)}}^{2}}{\left(F\left(\left\|\frac{\mathcal{A} u_{n}}{|x|^{\frac{\beta}{2 *}(\beta)}}\right\|_{L^{2_{s}^{*}(\beta)}},\left\|\frac{\mathcal{A} v_{n}}{|x|^{\frac{\beta}{2_{s}^{*}(\beta)}}}\right\|_{L_{s}^{2_{s}^{*}(\beta)}}\right)\right)^{\frac{2}{2_{s}^{*}(\beta)}}} \\
& \geq M_{F}^{-1} \Lambda(s, \beta) \text {. }
\end{aligned}
$$

Passing to the limit in the above inequality, we have

$$
S_{F}(s, \beta) \geq M_{F}^{-1} \Lambda(s, \beta) \text {. }
$$

Hence, (2.6) and (2.7) given the proof of Theorem 1. 
For the best constant $\Lambda(s, \beta)$, from [6,7], we know that, for all $0<s<1$ and $0 \leq \gamma<\gamma_{H}$, the best constant $\Lambda(s, \beta)$ is achieved by the form

$$
U_{\varepsilon}(x)=\varepsilon^{-\frac{N-2 s}{2}} U(x / \varepsilon), \quad \forall \varepsilon>0,
$$

where $U \in C^{1}\left(\mathbb{R}^{N} \backslash\{0\}\right)$ is a positive, radially symmetric, radially decreasing ground state solution of the limit problem:

$$
\begin{cases}(-\Delta)^{s} u-\gamma \frac{u}{|x|^{2 s}}=\frac{u^{2_{s}^{*}(\beta)-1}}{|x|^{\beta}}, & \text { in } \mathbb{R}^{N}, \\ u \geq 0, \quad u \neq \equiv 0, & \text { in } \mathbb{R}^{N} .\end{cases}
$$

Moreover, at zero and infinity, the solution $U$ satisfies

$$
\lim _{|x| \rightarrow 0}|x|^{a(\gamma)} U(x)=\lambda_{0}>0 \text { and } \lim _{|x| \rightarrow \infty}|x|^{b(\gamma)} U(x)=\lambda_{\infty}>0,
$$

where $a(\gamma)$ and $b(\gamma)$ are solutions of the equation

$$
\Psi_{N, s}(\beta)=2^{2 s} \frac{\Gamma\left(\frac{N-\beta}{2}\right) \Gamma\left(\frac{2 s+\beta}{2}\right)}{\Gamma\left(\frac{N-2 s-\beta}{2}\right) \Gamma\left(\frac{\beta}{2}\right)}-\gamma=0
$$

and satisfy $0 \leq a(\gamma)<\frac{N-2 s}{2}<b(\gamma) \leq N-2 s$. By a direct computation, we get

$\int_{\mathbb{R}^{N}} \int_{\mathbb{R}^{N}} \frac{\left|U_{\varepsilon}(x)-U_{\varepsilon}(y)\right|^{2}}{|x-y|^{N+2 s}} d x d y-\gamma \int_{\mathbb{R}^{N}} \frac{\left|U_{\varepsilon}\right|^{2}}{|x|^{2 s}} d x=\int_{\mathbb{R}^{N}} \frac{\left|U_{\varepsilon}\right|^{2_{s}^{*}(\beta)}}{|x|^{\beta}} d x=\Lambda(s, \beta)^{\frac{2_{s}^{*}(\beta)}{2_{s}^{*}(\beta)-2}}$.

Take $\rho \in\left(0, r_{0}\right)$ small enough such that $B_{\rho}(0) \subset \Omega, B_{\rho}(0)=\left\{x \in \mathbb{R}^{N}\right.$ : $|x|<\rho\}$, where $r_{0}$ be given in $\left(\mathcal{H}_{2}\right)$. Choose the radial cut-off function $\varphi \in$ $C_{0}^{\infty}(\Omega)$ such that $0 \leq \varphi(x) \leq 1$ if $B_{\rho}(0), \varphi(x)=1$ in $B_{\frac{\rho}{2}}(0)$ and $\varphi(x)=0$ if $B_{\rho}(0)^{c}$. Set

$$
u_{\varepsilon}(x)=\varphi(x) U_{\varepsilon}(x), \quad \forall \varepsilon>0 .
$$

Proposition 1. (See [20], Proposition 2.3)Assume that $0<s<1,0 \leq \gamma<$ $\gamma_{H}, 0 \leq \alpha, \beta<2 s$ and $1 \leq q<2_{s}^{*}(\alpha)$. Then, the following estimates hold as $\varepsilon \rightarrow 0^{+}:$

$$
\begin{aligned}
& \left\|u_{\varepsilon}\right\|_{\gamma}^{2}=\Lambda(s, \beta)^{\frac{N-\beta}{2 s-\beta}}+O\left(\varepsilon^{2 b(\gamma)+2 s-N}\right) ; \\
& \int_{\Omega} \frac{\left|u_{\varepsilon}\right|^{2_{s}^{*}(\beta)}}{|x|^{\beta}} d x=\Lambda(s, \beta)^{\frac{N-\beta}{2 s-\beta}}+O\left(\varepsilon^{2_{s}^{*}(\beta) b(\gamma)+\beta-N}\right) ; \\
& \int_{\Omega} \frac{\left|u_{\varepsilon}\right|^{q}}{|x|^{\alpha}} d x= \begin{cases}C \varepsilon^{N-\alpha-\frac{q(N-2 s)}{2}}, & \text { if } q>(N-\alpha) / b(\gamma), \\
C \varepsilon^{N-\alpha-\frac{q(N-2 s)}{2}}|\ln \varepsilon|, & \text { if } q=(N-\alpha) / b(\gamma), \\
C \varepsilon^{q\left(b(\gamma)-\frac{N-2 s}{2}\right.}, & \text { if } q<(N-\alpha) / b(\gamma) .\end{cases}
\end{aligned}
$$




\section{The Palais-Smale condition}

In this section, we show that the functional $I_{\lambda, \mu}$ satisfies $(P S)_{c}$ conditions.

Definition 1. Let $c \in \mathbb{R}, W$ be a Banach space and $I_{\lambda, \mu} \in C^{1}(W, \mathbb{R})$. Then $\left\{\left(u_{n}, v_{n}\right)\right\}$ is a $(P S)_{c}$ sequence in $W$ for $I_{\lambda, \mu}$ if $I_{\lambda, \mu}\left(u_{n}, v_{n}\right)=c+o_{n}(1)$ and $I_{\lambda, \mu}^{\prime}\left(u_{n}, v_{n}\right)=o_{n}(1)$ strongly in $W^{-1}$ as $n \rightarrow \infty$. We say that $I_{\lambda, \mu}$ satisfies the $(P S)_{c}$ condition if any $(P S)_{c}$ sequence $\left\{\left(u_{n}, v_{n}\right)\right\}$ for $I_{\lambda, \mu}$ admits a convergent subsequence.

By the Hölder and Sobolev-Hardy inequalities, for all $u \in X_{0}^{s}(\Omega)$, we get

$$
\begin{aligned}
\int_{\Omega} \frac{f(x)|u|^{q}}{|x|^{\alpha}} d x & =\int_{\Omega} \frac{|u|^{q}}{|x|^{\frac{q}{2_{s}^{*}(\alpha)}}} \cdot \frac{f(x)}{|x|^{\left(1-\frac{q}{2_{s}^{*}(\alpha)}\right) \alpha}} d x \\
& \leq\left(\int_{\Omega} \frac{|f|^{\frac{2_{s}^{*}(\alpha)}{2_{s}^{*}(\alpha)-q}}}{|x|^{\alpha}} d x\right)^{\frac{2_{s}^{*}(\alpha)-q}{2_{s}^{*}(\alpha)}}\left(\int_{\Omega} \frac{|u|^{2_{s}^{*}(\alpha)}}{|x|^{\alpha}} d x\right)^{\frac{q}{2_{s}^{*}(\alpha)}} \\
& =\|f\|{ }_{L^{\frac{2_{s}^{*}(\alpha)}{2_{s}^{*}(\alpha)-q}}\left(\Omega,|x|^{-\alpha}\right)} \Lambda(s, \alpha)^{-\frac{q}{2}}\|u\|_{\gamma}^{q} .
\end{aligned}
$$

Then,

$$
\begin{aligned}
Q_{\lambda, \mu} & (u, v) \leq \Lambda(s, \alpha)^{-\frac{q}{2}}\left(\lambda\|f\|_{L^{p}\left(\Omega,|x|^{-\alpha}\right)}\|u\|_{\gamma}^{q}+\mu\|g\|_{L^{p}\left(\Omega,|x|^{-\alpha}\right)}\|v\|_{\gamma}^{q}\right) \\
= & \left(\left[\frac{2}{q}\left(\frac{1}{2}-\frac{1}{2_{s}^{*}(\beta)}\right)\left(\frac{1}{q}-\frac{1}{2_{s}^{*}(\beta)}\right)^{-1}\right]^{\frac{q}{2}}\|u\|_{\gamma}^{q}\right) \\
& \times\left(\left[\frac{2}{q}\left(\frac{1}{2}-\frac{1}{2_{s}^{*}(\beta)}\right)\left(\frac{1}{q}-\frac{1}{2_{s}^{*}(\beta)}\right)^{-1}\right]^{-\frac{q}{2}} \Lambda(s, \alpha)^{-\frac{q}{2}} \lambda\|f\|_{L^{p}\left(\Omega,|x|^{-\alpha}\right)}\right) \\
& +\left(\left[\frac{2}{q}\left(\frac{1}{2}-\frac{1}{2_{s}^{*}(\beta)}\right)\left(\frac{1}{q}-\frac{1}{2_{s}^{*}(\beta)}\right)^{-1}\right]^{\frac{q}{2}}\|v\|_{\gamma}^{q}\right) \\
& \times\left(\left[\frac{2}{q}\left(\frac{1}{2}-\frac{1}{2_{s}^{*}(\beta)}\right)\left(\frac{1}{q}-\frac{1}{2_{s}^{*}(\beta)}\right)^{-1}\right]^{-\frac{q}{2}} \Lambda(s, \alpha)^{-\frac{q}{2}} \mu\|g\|_{L^{p}\left(\Omega,|x|^{-\alpha}\right)}\right) \\
\leq & \left(\frac{1}{2}-\frac{1}{2_{s}^{*}(\beta)}\right)\left(\frac{1}{q}-\frac{1}{2_{s}^{*}(\beta)}\right)^{-1}\|(u, v)\|_{W}^{2} \\
& +C_{*}\left(\left(\lambda\|f\|_{L^{p}\left(\Omega,|x|^{-\alpha}\right)}\right)^{\frac{2}{2-q}}+\left(\mu\|g\|_{L^{p}\left(\Omega,|x|^{-\alpha}\right)}\right)^{\frac{2}{2-q}}\right),
\end{aligned}
$$

where

$$
C_{*}=\frac{2-q}{2}\left(\frac{2_{s}^{*}(\beta)-q}{2_{s}^{*}(\beta)-2}\right)^{\frac{q}{2-q}} \Lambda(s, \alpha)^{-\frac{q}{2-q}}>0 .
$$

Lemma 2. If $\left\{\left(u_{n}, v_{n}\right)\right\}$ is a $(P S)_{c}$ sequence for $I_{\lambda, \mu}$ with $\left(u_{n}, v_{n}\right) \rightarrow(u, v)$ weakly in $W$. Then, $I_{\lambda, \mu}^{\prime}(u, v)=0$ and there exists a positive constant $C_{0}$ depending on $q, s, \alpha, \beta$ and $N$ such that

$$
I_{\lambda, \mu}(u, v) \geq-C_{0}\left(\left(\lambda\|f\|_{L^{p}\left(\Omega,|x|^{-\alpha}\right)}\right)^{\frac{2}{2-q}}+\left(\mu\|g\|_{L^{p}\left(\Omega,|x|^{-\alpha}\right)}\right)^{\frac{2}{2-q}}\right) .
$$

Proof. Since $\left\{\left(u_{n}, v_{n}\right)\right\}$ is a $(P S)_{c}$ sequence for $I_{\lambda, \mu}$ with $\left(u_{n}, v_{n}\right) \rightarrow(u, v)$ in $W$, it is easy to check that $I_{\lambda, \mu}^{\prime}(u, v)=0$. In particular, we get that

$$
\left\langle I_{\lambda, \mu}^{\prime}(u, v),(u, v)\right\rangle=0
$$


namely

$$
\|(u, v)\|_{W}^{2}=\int_{\Omega} \frac{F(u, v)}{|x|^{\beta}} d x+Q_{\lambda, \mu}(u, v) .
$$

Then, from (3.2), we have

$$
\begin{aligned}
I_{\lambda, \mu}(u, v) & =\left(\frac{1}{2}-\frac{1}{2_{s}^{*}(\beta)}\right)\|(u, v)\|_{W}^{2}-\left(\frac{1}{q}-\frac{1}{2_{s}^{*}(\beta)}\right) Q_{\lambda, \mu}(u, v) \\
& \geq-\left(\frac{1}{q}-\frac{1}{2_{s}^{*}(\beta)}\right) C_{*}\left(\left(\lambda\|f\|_{\left.L^{p}\left(\Omega,|x|^{-\alpha}\right)\right)^{\frac{2}{2-q}}}+\left(\mu\|g\|_{\left.L^{p}\left(\Omega,|x|^{-\alpha}\right)\right)^{\frac{2}{2-q}}}\right)\right.\right. \\
& =-C_{0}\left(\left(\lambda\|f\|_{L^{p}\left(\Omega,|x|^{-\alpha}\right)}\right)^{\frac{2}{2-q}}+\left(\mu\|g\|_{L^{p}\left(\Omega,|x|^{-\alpha}\right)}\right)^{\frac{2}{2-q}}\right)
\end{aligned}
$$

where $C_{0}=\frac{\left(2_{s}^{*}(\beta)-q\right)(2-q)}{2 q 2_{s}^{*}(\beta)}\left(\frac{2_{s}^{*}(\beta)-q}{2_{s}^{*}(\beta)-2}\right)^{\frac{q}{2-q}} \Lambda(s, \alpha)^{-\frac{q}{2-q}}$ is a positive constant depending on $q, s, \alpha, \beta$ and $N$.

Lemma 3. $I_{\lambda, \mu}$ satisfies the $(P S)_{c}$ condition for all $c<c_{\infty}$, where

$$
\begin{aligned}
c_{\infty}:= & \frac{2 s-\beta}{2(N-\beta)} S_{F}(s, \beta)^{\frac{2_{s}^{*}(\beta)}{2_{s}^{*}(\beta)-2}} \\
& -C_{0}\left(\left(\lambda\|f\|_{L^{p}\left(\Omega,|x|^{-\alpha}\right)}\right)^{\frac{2}{2-q}}+\left(\mu\|g\|_{L^{p}\left(\Omega,|x|^{-\alpha}\right)}\right)^{\frac{2}{2-q}}\right) .
\end{aligned}
$$

Proof. Let $\left\{\left(u_{n}, v_{n}\right)\right\}$ be a $(P S)_{c}$ sequence for $c \in\left(-\infty, c_{\infty}\right)$. Similarly to the proof of [13, Lemma 2.3], it is easy to see that $\left\{\left(u_{n}, v_{n}\right)\right\}$ is bounded in $W$. Then, there exist a subsequence still denoted by $\left\{\left(u_{n}, v_{n}\right)\right\}$ and $(u, v) \in W$ such that $\left(u_{n}, v_{n}\right) \rightarrow(u, v)$ weakly in $W$, and

$$
\left\{\begin{array}{l}
u_{n} \rightarrow u, \quad v_{n} \rightarrow v \text { weakly in } L_{s}^{2_{s}^{*}(\beta)}\left(\Omega,|x|^{-\beta} d x\right) \\
u_{n} \rightarrow u, \quad v_{n} \rightarrow v \text { strongly in } L^{q}\left(\Omega,|x|^{-\alpha} d x\right), \forall 1 \leq q<2_{s}^{*}(\alpha), \\
u_{n}(x) \rightarrow u(x), \quad v_{n}(x) \rightarrow v(x) \text { a.e. in } \Omega
\end{array}\right.
$$

Hence, from (3.4), it is easy to verify that $I_{\lambda, \mu}^{\prime}(u, v)=0$ and

$$
Q_{\lambda, \mu}\left(u_{n}, v_{n}\right)=Q_{\lambda, \mu}(u, v)+o_{n}(1) \quad(n \rightarrow \infty) .
$$

Set $\tilde{u}_{n}=u_{n}-u, \tilde{v}_{n}=v_{n}-v$. By Brézis-Lieb lemma [19], we get

$$
\left\|\left(u_{n}, v_{n}\right)\right\|_{W}^{2}=\|(u, v)\|_{W}^{2}+\left\|\left(\tilde{u}_{n}, \tilde{v}_{n}\right)\right\|_{W}^{2}+o_{n}(1)
$$

By the same methods as in [4, Lemma 8], we obtain

$$
\int_{\Omega} \frac{F\left(u_{n}, v_{n}\right)}{|x|^{\beta}} d x=\int_{\Omega} \frac{F(u, v)}{|x|^{\beta}} d x+\int_{\Omega} \frac{F\left(\tilde{u}_{n}, \tilde{v}_{n}\right)}{|x|^{\beta}} d x+o_{n}(1) .
$$

Using (3.5), (3.6) and (3.7), we have

$$
c=\frac{1}{2}\left\|\left(\tilde{u}_{n}, \tilde{v}_{n}\right)\right\|_{W}^{2}-\frac{1}{2_{s}^{*}(\beta)} \int_{\Omega} \frac{F\left(\tilde{u}_{n}, \tilde{v}_{n}\right)}{|x|^{\beta}} d x+I_{\lambda, \mu}(u, v)+o_{n}(1)
$$


and

$$
o_{n}(1)=\left\|\left(\tilde{u}_{n}, \tilde{v}_{n}\right)\right\|_{W}^{2}-\int_{\Omega} \frac{F\left(\tilde{u}_{n}, \tilde{v}_{n}\right)}{|x|^{\beta}} d x .
$$

Thus, we may assume that

$$
\int_{\Omega} \frac{F\left(\tilde{u}_{n}, \tilde{v}_{n}\right)}{|x|^{\beta}} d x \rightarrow l, \quad\left\|\left(\tilde{u}_{n}, \tilde{v}_{n}\right)\right\|_{W}^{2} \rightarrow l \geq 0 \quad \text { as } n \rightarrow \infty .
$$

If $l=0$, the proof is completed. Assume that $l>0$, then from (3.9) we have

$$
S_{F}(s, \beta) l^{\frac{2}{2 *(\beta)}}=S_{F}(s, \beta)\left(\lim _{n \rightarrow \infty} \int_{\Omega} \frac{F\left(\tilde{u}_{n}, \tilde{v}_{n}\right)}{|x|^{\beta}} d x\right)^{\frac{2}{2_{s}^{*}(\beta)}} \leq \lim _{n \rightarrow \infty}\left\|\left(\tilde{u}_{n}, \tilde{v}_{n}\right)\right\|_{W}^{2}=l,
$$

which implies that $l \geq S_{F}(s, \beta)^{\frac{2_{s}^{*}(\beta)}{2 *(\beta)-2}}$. Hence, from (3.8) and Lemma 2, we have

$$
\begin{aligned}
c= & I_{\lambda, \mu}\left(u_{n}, v_{n}\right)+o_{n}(1)=\left(\frac{1}{2}-\frac{1}{2_{s}^{*}(\beta)}\right) l+I_{\lambda, \mu}(u, v)+o_{n}(1) \geq \frac{2 s-\beta}{2(N-\beta)} \\
& \times S_{F}(s, \beta)^{\frac{2_{s}^{*}(\beta)}{2(\beta)-2}}-C_{0}\left(\left(\lambda\|f\|_{L^{p}\left(\Omega,|x|^{-\alpha}\right)}\right)^{\frac{2}{2-q}}+\left(\mu\|g\|_{L^{p}\left(\Omega,|x|^{-\alpha}\right)}\right)^{\frac{2}{2-q}}\right),
\end{aligned}
$$

which contradicts $c<c_{\infty}$. The proof is completed.

\section{Nehari manifold}

Since $I_{\lambda, \mu}$ is not bounded below on $W$, we need to study $I_{\lambda, \mu}$ on the Nehari manifold

$$
\mathcal{N}_{\lambda, \mu}=\left\{(u, v) \in W \backslash\{(0,0)\}:\left\langle I_{\lambda, \mu}^{\prime}(u, v),(u, v)\right\rangle=0\right\} .
$$

Note that $\mathcal{N}_{\lambda, \mu}$ contains all nonzero solution of $(1.1)$, and $(u, v) \in \mathcal{N}_{\lambda, \mu}$ if and only if

$$
\|(u, v)\|_{W}^{2}-\int_{\Omega} \frac{F(u, v)}{|x|^{\beta}} d x-Q_{\lambda, \mu}(u, v)=0 .
$$

Lemma 4. $I_{\lambda, \mu}$ is coercive and bounded below on $\mathcal{N}_{\lambda, \mu}$.

Proof. $\quad$ Let $(u, v) \in \mathcal{N}_{\lambda, \mu}$, by (3.1), the Sobolev inequality and Hölder inequality, we find

$$
Q_{\lambda, \mu}(u, v) \leq C_{1}\|(u, v)\|_{W}^{q},
$$

where

$$
C_{1}=\left[\left(\lambda\|f\|_{L^{p}\left(\Omega,|x|^{-\alpha}\right)}\right)^{\frac{2}{2-q}}+\left(\mu\|g\|_{L^{p}\left(\Omega,|x|^{-\alpha}\right)}\right)^{\frac{2}{2-q}}\right]^{\frac{2-q}{2}} \Lambda(s, \alpha)^{-\frac{q}{2}}>0 .
$$

From (4.1) and (4.2), we get

$$
\begin{aligned}
I_{\lambda, \mu}(u, v) & =\left(\frac{1}{2}-\frac{1}{2_{s}^{*}(\beta)}\right)\|(u, v)\|_{W}^{2}-\left(\frac{1}{q}-\frac{1}{2_{s}^{*}(\beta)}\right) Q_{\lambda, \mu}(u, v) \\
& \geq \frac{2_{s}^{*}(\beta)-2}{22_{s}^{*}(\beta)}\|(u, v)\|_{W}^{2}-\frac{2_{s}^{*}(\beta)-q}{q 2_{s}^{*}(\beta)} C_{1}\|(u, v)\|_{W}^{q} .
\end{aligned}
$$


As $1 \leq q<2, I_{\lambda, \mu}$ is coercive and bounded below on $\mathcal{N}_{\lambda, \mu}$.

Define $\Psi_{\lambda, \mu}(u, v):=\left\langle I_{\lambda, \mu}^{\prime}(u, v),(u, v)\right\rangle$, then for all $(u, v) \in \mathcal{N}_{\lambda, \mu}$, we get

$$
\begin{aligned}
\left\langle\Psi_{\lambda, \mu}^{\prime}(u, v),(u, v)\right\rangle & =2\|(u, v)\|_{W}^{2}-q Q_{\lambda, \mu}(u, v)-2_{s}^{*}(\beta) \int_{\Omega} \frac{F(u, v)}{|x|^{\beta}} d x \\
& =(2-q)\|(u, v)\|_{W}^{2}-\left(2_{s}^{*}(\beta)-q\right) \int_{\Omega} \frac{F(u, v)}{|x|^{\beta}} d x \\
& =\left(2_{s}^{*}(\beta)-q\right) Q_{\lambda, \mu}(u, v)-\left(2_{s}^{*}(\beta)-2\right)\|(u, v)\|_{W}^{2} .
\end{aligned}
$$

We split $\mathcal{N}_{\lambda, \mu}$ into three parts:

$$
\begin{aligned}
& \mathcal{N}_{\lambda, \mu}^{+}=\left\{(u, v) \in \mathcal{N}_{\lambda, \mu}:\left\langle\Psi_{\lambda, \mu}^{\prime}(u, v),(u, v)\right\rangle>0\right\}, \\
& \mathcal{N}_{\lambda, \mu}^{0}=\left\{(u, v) \in \mathcal{N}_{\lambda, \mu}:\left\langle\Psi_{\lambda, \mu}^{\prime}(u, v),(u, v)\right\rangle=0\right\}, \\
& \mathcal{N}_{\lambda, \mu}^{-}=\left\{(u, v) \in \mathcal{N}_{\lambda, \mu}:\left\langle\Psi_{\lambda, \mu}^{\prime}(u, v),(u, v)\right\rangle<0\right\} .
\end{aligned}
$$

We now present some important properties of $\mathcal{N}_{\lambda, \mu}, \mathcal{N}_{\lambda, \mu}^{+}, \mathcal{N}_{\lambda, \mu}^{-}$.

Lemma 5. Assume that $\left(u_{0}, v_{0}\right)$ is a local minimizer for $I_{\lambda, \mu}$ on $\mathcal{N}_{\lambda, \mu}$ and $\left(u_{0}, v_{0}\right) \notin \mathcal{N}_{\lambda, \mu}^{0}$. Then $I_{\lambda, \mu}^{\prime}\left(u_{0}, v_{0}\right)=0$ in $W^{-1}$.

Proof. The proof is similar to that of [20, Lemma 3.4] and the details are omitted.

Lemma 6. $\mathcal{N}_{\lambda, \mu}^{0}=\emptyset$ for all $\lambda, \mu>0$ satisfy $0<\left(\lambda\|f\|_{L^{p}\left(\Omega,|x|^{-\alpha}\right)}\right)^{\frac{2}{2-q}}+$ $\left(\mu\|g\|_{L^{p}\left(\Omega,|x|^{-\alpha}\right)}\right)^{\frac{2}{2-q}}<\Lambda_{0}$, where $\Lambda_{0}$ is the same as that in (1.6).

Proof. We argue by contradiction. Assume that there exist $\lambda$ and $\mu>0$ with $0<\left(\lambda\|f\|_{L^{p}\left(\Omega,|x|^{-\alpha}\right)}\right)^{\frac{2}{2-q}}+\left(\mu\|g\|_{L^{p}\left(\Omega,|x|^{-\alpha}\right)}\right)^{\frac{2}{2-q}}<\Lambda_{0}$ such that $\mathcal{N}_{\lambda, \mu}^{0} \neq \emptyset$. Then, by (4.3) and (4.4), for $(u, v) \in \mathcal{N}_{\lambda, \mu}^{0}$, we have

$$
\|(u, v)\|_{W}^{2}=\frac{2_{s}^{*}(\beta)-q}{2-q} \int_{\Omega} \frac{F(u, v)}{|x|^{\beta}} d x,\|(u, v)\|_{W}^{2}=\frac{2_{s}^{*}(\beta)-q}{2_{s}^{*}(\beta)-2} Q_{\lambda, \mu}(u, v) .
$$

According to (2.3) and the Minkowshi inequality, we obtain that

$$
\begin{aligned}
& \int_{\Omega} \frac{F(u, v)}{|x|^{\beta}} d x \leq M_{F}^{\frac{2_{s}^{*}(\beta)}{2}} \int_{\Omega} \frac{\left(|u|^{2}+|v|^{2}\right)^{\frac{2_{s}^{*}(\beta)}{2}}}{|x|^{\beta}} d x \\
& \quad \leq M_{F}^{\frac{2_{s}^{*}(\beta)}{2}}\left[\left(\int_{\Omega} \frac{|u|^{2_{s}^{*}(\beta)}}{|x|^{\beta}} d x\right)^{\frac{2}{2_{s}^{*}(\beta)}}+\left(\int_{\Omega} \frac{|v|^{2_{s}^{*}(\beta)}}{|x|^{\beta}} d x\right)^{\frac{2_{s}^{2}(\beta)}{22_{s}^{*}(\beta)}}\right]^{\frac{2_{s}^{*}}{2}} \\
& \quad \leq M_{F}^{\frac{2_{s}^{*}(\beta)}{{ }^{2}}}\left(\Lambda(s, \beta)^{-1}\|u\|_{\gamma}^{2}+\Lambda(s, \beta)^{-1}\|v\|_{\gamma}^{2}\right)^{\frac{2_{s}^{*}(\beta)}{2}} \\
& =\left(\frac{\Lambda(s, \beta)}{M_{F}}\right)^{-\frac{2_{s}^{*}(\beta)}{2}}\left(\|u\|_{\gamma}^{2}+\|v\|_{\gamma}^{2}\right)^{\frac{2_{s}^{*}(\beta)}{2}}=S_{F}(s, \beta)^{-\frac{2_{s}^{*}(\beta)}{2}}\|(u, v)\|_{W}^{2_{s}^{*}(\beta)},
\end{aligned}
$$


which and (4.5) leads that

$$
\|(u, v)\|_{W} \geq\left(\frac{2-q}{2_{s}^{*}(\beta)-q} S_{F}(s, \beta)^{\frac{2_{s}^{*}(\beta)}{2}}\right)^{\frac{1}{2_{s}^{*}(\beta)-2}} .
$$

On the other hand, by (4.2) and (4.5), we find that

$$
\begin{aligned}
\|(u, v)\|_{W} \leq & \left(\frac{2_{s}^{*}(\beta)-q}{2_{s}^{*}(\beta)-2}\right)^{\frac{1}{2-q}} \Lambda(s, \alpha)^{-\frac{q}{2(2-q)}} \\
& \times\left(\left(\lambda\|f\|_{L^{p}\left(\Omega,|x|^{-\alpha}\right)}\right)^{\frac{2}{2-q}}+\left(\mu\|g\|_{L^{p}\left(\Omega,|x|^{-\alpha}\right)}\right)^{\frac{2}{2-q}}\right)^{\frac{1}{2}} .
\end{aligned}
$$

Consequently, (4.7) and (4.8) implies

$$
\left(\lambda\|f\|_{L^{p}\left(\Omega,|x|^{-\alpha}\right)}\right)^{\frac{2}{2-q}}+\left(\mu\|g\|_{L^{p}\left(\Omega,|x|^{-\alpha}\right)}\right)^{\frac{2}{2-q}} \geq \Lambda_{0},
$$

which contradicts to $0<\left(\lambda\|f\|_{L^{p}\left(\Omega,|x|^{-\alpha}\right)}\right)^{\frac{2}{2-q}}+\left(\mu\|g\|_{L^{p}\left(\Omega,|x|^{-\alpha}\right)}\right)^{\frac{2}{2-q}}<\Lambda_{0}$. This completes the proof of Lemma 6 .

By Lemmas 4 and 6 , for each $\lambda, \mu>0$ with $0<\left(\lambda\|f\|_{L^{p}\left(\Omega,|x|^{-\alpha}\right)}\right)^{\frac{2}{2-q}}+$ $\left(\mu\|g\|_{L^{p}\left(\Omega,|x|^{-\alpha}\right)}\right)^{\frac{2}{2-q}}<\Lambda_{0}$, we can write $\mathcal{N}_{\lambda, \mu}=\mathcal{N}_{\lambda, \mu}^{+} \cup \mathcal{N}_{\lambda, \mu}^{-}$. Define

$c_{\lambda, \mu}=\inf _{(u, v) \in \mathcal{N}_{\lambda, \mu}} I_{\lambda, \mu}(u, v), c_{\lambda, \mu}^{+}=\inf _{(u, v) \in \mathcal{N}_{\lambda, \mu}^{+}} I_{\lambda, \mu}(u, v), c_{\lambda, \mu}^{-}=\inf _{(u, v) \in \mathcal{N}_{\lambda, \mu}^{-}} I_{\lambda, \mu}(u, v)$.

Then, we have the following results.

Lemma 7. The following results hold.

(1) If $0<\left(\lambda\|f\|_{L^{p}\left(\Omega,|x|^{-\alpha}\right)}\right)^{\frac{2}{2-q}}+\left(\mu\|g\|_{L^{p}\left(\Omega,|x|^{-\alpha}\right)}\right)^{\frac{2}{2-q}}<\Lambda_{0}$, then $c_{\lambda, \mu} \leq$ $c_{\lambda, \mu}^{+}<0$.

(2) There exists

$$
\Lambda_{1}:=\left(\frac{q\left(2_{s}^{*}(\beta)-2\right)}{2\left(2_{s}^{*}(\beta)-q\right)}\right)^{\frac{2}{2-q}} \Lambda(s, \alpha)^{\frac{q}{2-q}} S_{F}(s, \beta)^{\frac{2_{s}^{*}(\beta)}{2_{s}^{*}(\beta)-2}}\left(\frac{2-q}{2_{s}^{*}(\beta)-q}\right)^{\frac{2}{2_{s}^{*}(\beta)-2}}
$$

such that for all $\lambda, \mu>0$ with

$$
0<\left(\lambda\|f\|_{L^{p}\left(\Omega,|x|^{-\alpha}\right)}\right)^{\frac{2}{2-q}}+\left(\mu\|g\|_{L^{p}\left(\Omega,|x|^{-\alpha}\right)}\right)^{\frac{2}{2-q}}<\Lambda_{1},
$$

then $c_{\lambda, \mu}^{-} \geq c_{0}$ for some $c_{0}>0$.

Proof. (1) Suppose $(u, v) \in \mathcal{N}_{\lambda, \mu}^{+}$. By (4.3), we get

$$
(2-q)\|(u, v)\|_{W}^{2}>\left(2_{s}^{*}(\beta)-q\right) \int_{\Omega} \frac{F(u, v)}{|x|^{\beta}} d x .
$$


According to (4.1) and (4.9), we get

$$
\begin{aligned}
I_{\lambda, \mu}(u, v) & =\left(\frac{1}{2}-\frac{1}{q}\right)\|(u, v)\|_{W}^{2}-\left(\frac{1}{2_{s}^{*}(\beta)}-\frac{1}{q}\right) \int_{\Omega} \frac{F(u, v)}{|x|^{\beta}} d x \\
& \leq\left[\left(\frac{1}{2}-\frac{1}{q}\right)+\left(\frac{1}{q}-\frac{1}{2_{s}^{*}(\beta)}\right) \frac{2-q}{2_{s}^{*}(\beta)-q}\right]\|(u, v)\|_{W}^{2} \\
& =-\frac{(2-q)\left(2_{s}^{*}(\beta)-2\right)}{2 q 2_{s}^{*}(\beta)}\|(u, v)\|_{W}^{2}<0 .
\end{aligned}
$$

Then, by the definition of $c_{\lambda, \mu}, c_{\lambda, \mu}^{+}$, we can deduce that $c_{\lambda, \mu} \leq c_{\lambda, \mu}^{+}<0$.

(2) Suppose $(u, v) \in \mathcal{N}_{\lambda, \mu}^{-}$. From (4.3), it follows that

$$
(2-q)\|(u, v)\|_{W}^{2}<\left(2_{s}^{*}(\beta)-q\right) \int_{\Omega} \frac{F(u, v)}{|x|^{\beta}} d x .
$$

This and (4.6) yield

$$
\|(u, v)\|_{W}>\left(\frac{2-q}{2_{s}^{*}(\beta)-q}\right)^{\frac{1}{2_{s}^{*}(\beta)-2}} S_{F}(s, \beta)^{\frac{2_{s}^{*}(\beta)}{2\left(2_{s}^{*}(\beta)-2\right)}} .
$$

By the proof of Lemma 4 and (4.10), we infer that

$$
\begin{aligned}
& I_{\lambda, \mu}(u, v) \\
& \geq\|(u, v)\|_{W}^{q}\left[\left(\frac{1}{2}-\frac{1}{2_{s}^{*}(\beta)}\right)\|(u, v)\|_{W}^{2-q}-\left(\frac{2_{s}^{*}(\beta)-q}{q 2_{s}^{*}(\beta)}\right)\right. \\
& \left.\times\left(\left(\lambda\|f\|_{L^{p}\left(\Omega,|x|^{-\alpha}\right)}\right)^{\frac{2}{2-q}}+\left(\mu\|g\|_{L^{p}\left(\Omega,|x|^{-\alpha}\right)}\right)^{\frac{2}{2-q}}\right)^{\frac{2-q}{2}} \Lambda(s, \alpha)^{-\frac{q}{2}}\right] \\
& >\|(u, v)\|_{W}^{q}\left[\frac{2_{s}^{*}(\beta)-2}{22_{s}^{*}(\beta)}\left(\frac{2-q}{2_{s}^{*}(\beta)-q}\right)^{\frac{2-q}{22_{s}^{*}(\beta)-2}} S_{F}(s, \beta)^{\frac{2_{s}^{*}(\beta)(2-q)}{2\left(2_{s}^{*}(\beta)-2\right)}}-\left(\frac{2_{s}^{*}(\beta)-q}{q 2_{s}^{*}(\beta)}\right)\right. \\
& \left.\times\left(\left(\lambda\|f\|_{L^{p}\left(\Omega,|x|^{-\alpha}\right)}\right)^{\frac{2}{2-q}}+\left(\mu\|g\|_{L^{p}\left(\Omega,|x|^{-\alpha}\right)}\right)^{\frac{2}{2-q}}\right)^{\frac{2-q}{2}} \Lambda(s, \alpha)^{-\frac{q}{2}}\right] .
\end{aligned}
$$

Then, if $\left(\lambda\|f\|_{L^{p}\left(\Omega,|x|^{-\alpha}\right)}\right)^{\frac{2}{2-q}}+\left(\mu\|g\|_{L^{p}\left(\Omega,|x|^{-\alpha}\right)}\right)^{\frac{2}{2-q}}<\Lambda_{1}$, we get $I_{\lambda, \mu}(u, v) \geq$ $c_{0}$ for all $(u, v) \in \mathcal{N}_{\lambda, \mu}^{-}$, where $c_{0}=c(q, s, \alpha, \beta, N)$ is a positive constant.

For $t>0$, we define the fibering maps $\Phi_{u, v}(t)=I_{\lambda, \mu}(t u, t v)$. Then,

$$
\Phi_{u, v}^{\prime}(t)=t\|(u, v)\|_{W}^{2}-t^{2_{s}^{*}(\beta)-1} \int_{\Omega} \frac{F(u, v)}{|x|^{\beta}} d x-t^{q-1} Q_{\lambda, \mu}(u, v) .
$$

For $(u, v) \in \mathcal{N}_{\lambda, \mu}$, we get $\Phi_{u, v}^{\prime}(1)=\left\langle I_{\lambda, \mu}^{\prime}(u, v),(u, v)\right\rangle$, which implies that $(u, v) \in \mathcal{N}_{\lambda, \mu}$ if and only if $\Phi_{u, v}^{\prime}(1)=0$, and more generally $(t u, t v) \in \mathcal{N}_{\lambda, \mu}$ if and only if $\Phi_{u, v}^{\prime}(t)=0$. That is, the elements in $\mathcal{N}_{\lambda, \mu}$ correspond to stationary points of the fibering maps $\Phi_{u, v}(t)$.

For each $(u, v) \in W$ with $\int_{\Omega} \frac{F(u, v)}{|x|^{\beta}} d x>0$, set

$$
t_{\max }=\left(\frac{(2-q)\|(u, v)\|_{W}^{2}}{\left(2_{s}^{*}(\beta)-q\right) \int_{\Omega} \frac{F(u, v)}{|x|^{\beta}} d x}\right)^{\frac{1}{2_{s}^{*}(\beta)-2}}>0 .
$$

Then, the following lemma holds. 
Lemma 8. If $0<\left(\lambda\|f\|_{L^{p}\left(\Omega,|x|^{-\alpha}\right)}\right)^{\frac{2}{2-q}}+\left(\mu\|g\|_{L^{p}\left(\Omega,|x|^{-\alpha}\right)}\right)^{\frac{2}{2-q}}<\Lambda_{0}$, then for every $(u, v) \in W \backslash\{(0,0)\}$ with $\int_{\Omega} \frac{F(u, v)}{|x|^{\beta}} d x>0$ and $Q_{\lambda, \mu}(u, v)>0$, there exist unique $t_{1}$ and $t_{2}>0$ such that $\left(t_{1} u, t_{1} v\right) \in \mathcal{N}_{\lambda, \mu}^{+},\left(t_{2} u, t_{2} v\right) \in \mathcal{N}_{\lambda, \mu}^{-}$. Moreover, we have $0<t_{1}<t_{\max }<t_{2}, I_{\lambda, \mu}\left(t_{1} u, t_{1} v\right)=\inf _{t \in\left[0, t_{\max }\right]} I_{\lambda, \mu}(t u, t v)$ and $I_{\lambda, \mu}\left(t_{2} u, t_{2} v\right)=\sup _{t \in[0, \infty)} I_{\lambda, \mu}(t u, t v)$.

Proof. The proof is similar to [20, Lemma 3.2] and the details are omitted.

\section{Proof of Theorem 2}

Before giving the proof of Theorem 2, we need the following lemma.

Lemma 9. Suppose $\left(\mathcal{H}_{0}\right)-\left(\mathcal{H}_{2}\right)$ and $\left(\mathcal{F}_{0}\right)-\left(\mathcal{F}_{2}\right)$ hold. The following facts hold.

(i) If $0<\left(\lambda\|f\|_{L^{p}\left(\Omega,|x|^{-\alpha}\right)}\right)^{\frac{2}{2-q}}+\left(\mu\|g\|_{L^{p}\left(\Omega,|x|^{-\alpha}\right)}\right)^{\frac{2}{2-q}}<\Lambda_{0}$, then there exists $a(P S)_{c_{\lambda, \mu}}$-sequence $\left\{\left(u_{n}, v_{n}\right)\right\} \subset \mathcal{N}_{\lambda, \mu}$ for $I_{\lambda, \mu}$.

(ii) If $0<\left(\lambda\|f\|_{L^{p}\left(\Omega,|x|^{-\alpha}\right)}\right)^{\frac{2}{2-q}}+\left(\mu\|g\|_{L^{p}\left(\Omega,|x|^{-\alpha}\right)}\right)^{\frac{2}{2-q}}<\Lambda_{1}$, then there exists $a(P S)_{c_{\lambda, \mu}^{-}}$-sequence $\left\{\left(u_{n}, v_{v}\right)\right\} \subset \mathcal{N}_{\lambda, \mu}^{-}$for $I_{\lambda, \mu}$.

Proof. The proof is almost the same as in [18, Proposition 3.8] and the details are omitted.

Now, we establish the existence of a local minimizer for $I_{\lambda, \mu}$ on $\mathcal{N}_{\lambda, \mu}^{+}$.

Theorem 3. Assume that $\left(\mathcal{H}_{0}\right)-\left(\mathcal{H}_{2}\right)$ and $\left(F_{0}\right)-\left(F_{2}\right)$. If

$$
0<\left(\lambda\|f\|_{L^{p}\left(\Omega,|x|^{-\alpha}\right)}\right)^{\frac{2}{2-q}}+\left(\mu\|g\|_{L^{p}\left(\Omega,|x|^{-\alpha}\right)}\right)^{\frac{2}{2-q}}<\Lambda_{0},
$$

then $I_{\lambda, \mu}$ has a minimizer $\left(u_{1}, v_{1}\right) \in \mathcal{N}_{\lambda, \mu}^{+}$such that $\left(u_{1}, v_{1}\right)$ is a positive solution of (1.1) and $I_{\lambda, \mu}\left(u_{1}, v_{1}\right)=c_{\lambda, \mu}=c_{\lambda, \mu}^{+}<0$.

Proof. By Lemma 9 (i), there exists a minimizing sequence $\left\{\left(u_{n}, v_{n}\right)\right\} \subset \mathcal{N}_{\lambda, \mu}$ such that

$$
I_{\lambda, \mu}\left(u_{n}, v_{n}\right)=c_{\lambda, \mu}+o_{n}(1) \quad \text { and } \quad I_{\lambda, \mu}^{\prime}\left(u_{n}, v_{n}\right)=o_{n}(1) .
$$

Since $I_{\lambda, \mu}$ is coercive on $\mathcal{N}_{\lambda, \mu}$, we get that $\left\{\left(u_{n}, v_{n}\right)\right\}$ is bounded in $W$. Passing to a subsequence, still denoted by $\left\{\left(u_{n}, v_{n}\right)\right\}$, we can assume that there exists $\left(u_{1}, v_{1}\right) \in W$ such that $\left(u_{n}, v_{n}\right) \rightarrow\left(u_{1}, v_{1}\right)$ weakly in $W$ and

$$
\left\{\begin{array}{l}
u_{n} \rightarrow u_{1}, \quad v_{n} \rightarrow v_{1} \text { weakly in } L^{2_{s}^{*}(\beta)}\left(\Omega,|x|^{-\beta} d x\right), \\
u_{n} \rightarrow u_{1}, \quad v_{n} \rightarrow v_{1} \text { strongly in } L^{q}\left(\Omega,|x|^{-\alpha} d x\right), \quad \forall 1 \leq q<2_{\alpha}^{*}(s), \\
u_{n}(x) \rightarrow u_{1}(x), \quad v_{n}(x) \rightarrow v_{1}(x) \text { a.e. in } \Omega
\end{array}\right.
$$

This implies that

$$
Q_{\lambda, \mu}\left(u_{n}, v_{n}\right)=Q_{\lambda, \mu}\left(u_{1}, v_{1}\right)+o_{n}(1)
$$


First, we claim that $\left(u_{1}, v_{1}\right)$ is a nontrivial weak solution of (1.1). From (5.1), (5.2) and (5.3), it is easy to verify that $\left(u_{1}, v_{1}\right)$ is a weak solution of (1.1). Moreover, the fact $\left(u_{n}, v_{n}\right) \in \mathcal{N}_{\lambda, \mu}$ implies that

$$
Q_{\lambda, \mu}\left(u_{n}, v_{n}\right)=\frac{q\left(2_{s}^{*}(\beta)-2\right)}{2\left(2_{s}^{*}(\beta)-q\right)}\left\|\left(u_{n}, v_{n}\right)\right\|_{W}^{2}-\frac{q 2_{s}^{*}(\beta)}{2_{s}^{*}(\beta)-q} I_{\lambda, \mu}\left(u_{n}, v_{n}\right) .
$$

Let $n \rightarrow \infty$ in (5.4), by (5.3) and the fact that $c_{\lambda, \mu}<0$, we obtain

$$
Q_{\lambda, \mu}\left(u_{1}, v_{1}\right) \geq-\frac{q 2_{s}^{*}(\beta)}{2_{s}^{*}(\beta)-q} c_{\lambda, \mu}>0 .
$$

Thus, $\left(u_{1}, v_{1}\right) \in \mathcal{N}_{\lambda, \mu}$ is a nontrivial weak solution of (1.1).

Next, we prove that $\left(u_{n}, v_{n}\right) \rightarrow\left(u_{1}, v_{1}\right)$ strongly in $W$ and $I_{\lambda, \mu}\left(u_{1}, v_{1}\right)=$ $c_{\lambda, \mu}$. From the fact $\left(u_{1}, v_{1}\right) \in \mathcal{N}_{\lambda, \mu}$ and the Fatou's lemma, it follows that

$$
\begin{aligned}
c_{\lambda, \mu} & \leq I_{\lambda, \mu}\left(u_{1}, v_{1}\right)=\frac{2_{s}^{*}(\beta)-2}{22_{s}^{*}(\beta)}\left\|\left(u_{1}, v_{1}\right)\right\|_{W}^{2}-\frac{2_{s}^{*}(\beta)-q}{q 2_{s}^{*}(\beta)} Q_{\lambda, \mu}\left(u_{1}, v_{1}\right) \\
& \leq \lim _{n \rightarrow \infty}\left[\frac{2_{s}^{*}(\beta)-2}{22_{s}^{*}(\beta)}\left\|\left(u_{n}, v_{n}\right)\right\|_{W}^{2}-\frac{2_{s}^{*}(\beta)-q}{q 2_{s}^{*}(\beta)} Q_{\lambda, \mu}\left(u_{n}, v_{n}\right)\right] \\
& =\lim _{n \rightarrow \infty} I_{\lambda, \mu}\left(u_{n}, v_{n}\right)=c_{\lambda, \mu},
\end{aligned}
$$

which implies that $c_{\lambda, \mu}=I_{\lambda, \mu}\left(u_{1}, v_{1}\right)$ and $\lim _{n \rightarrow \infty}\left\|\left(u_{n}, v_{n}\right)\right\|_{W}^{2}=\left\|\left(u_{1}, v_{1}\right)\right\|_{W}^{2}$. Standard argument shows that $\left(u_{n}, v_{n}\right) \rightarrow\left(u_{1}, v_{1}\right)$ strongly in $W$.

Finally, we claim that $\left(u_{1}, v_{1}\right) \in \mathcal{N}_{\lambda, \mu}^{+}$. Otherwise, if $\left(u_{1}, v_{1}\right) \in \mathcal{N}_{\lambda, \mu}^{-}$, then by Lemma 8 , there exist unique $t_{1}^{+}$and $t_{1}^{-}>0$ such that $\left(t_{1}^{+} u_{1}, t_{1}^{+} v_{1}\right) \in \mathcal{N}_{\lambda, \mu}^{+}$ and $\left(t_{1}^{-} u_{1}, t_{1}^{-} v_{1}\right) \in \mathcal{N}_{\lambda, \mu}^{-}$. In particular, we have $t_{1}^{+}<t_{1}^{-}=1$. Since

$$
\frac{d}{d t} I_{\lambda, \mu}\left(t_{1}^{+} u_{1}, t_{1}^{+} v_{1}\right)=0, \quad \frac{d^{2}}{d t^{2}} I_{\lambda, \mu}\left(t_{1}^{+} u_{1}, t_{1}^{+} v_{1}\right)>0
$$

there exists $t_{1}^{*} \in\left(t_{1}^{+}, t_{1}^{-}\right)$such that $I_{\lambda, \mu}\left(t_{1}^{+} u_{1}, t_{1}^{+} v_{1}\right)<I_{\lambda, \mu}\left(t_{1}^{*} u_{1}, t_{1}^{*} v_{1}\right)$. By Lemma 8, we have

$$
I_{\lambda, \mu}\left(t_{1}^{+} u_{1}, t_{1}^{+} v_{1}\right)<I_{\lambda, \mu}\left(t_{1}^{*} u_{1}, t_{1}^{*} v_{1}\right) \leq I_{\lambda, \mu}\left(t_{1}^{-} u_{1}, t_{1}^{-} v_{1}\right)=I_{\lambda, \mu}\left(u_{1}, v_{1}\right),
$$

which contradicts $I_{\lambda, \mu}\left(u_{1}, v_{1}\right)=c_{\lambda, \mu}$. Moreover, from

$$
I_{\lambda, \mu}\left(u_{1}, v_{1}\right)=I_{\lambda, \mu}\left(\left|u_{1}\right|,\left|v_{1}\right|\right), \quad\left(\left|u_{1}\right|,\left|v_{1}\right|\right) \in \mathcal{N}_{\lambda, \mu}^{+}
$$

and the strong maximum principle [2], we conclude that $u_{1}, v_{1}>0$. Hence, $\left(u_{1}, v_{1}\right)$ is a positive solution for (1.1).

Proof of Theorem 2 (i). By Theorem 3, we obtain that for all $\lambda, \mu>0$ with $0<\left(\lambda\|f\|_{L^{p}\left(\Omega,|x|^{-\alpha}\right)}\right)^{\frac{2}{2-q}}+\left(\mu\|g\|_{L^{p}\left(\Omega,|x|^{-\alpha}\right)}\right)^{\frac{2}{2-q}}<\Lambda_{0}$, problem (1.1) has a positive solution $\left(u_{1}, v_{1}\right) \in \mathcal{N}_{\lambda, \mu}^{+}$. 
Remark 2. From Lemma 7 (i) and (4.2), for this positive solution $\left(u_{1}, v_{1}\right)$, we have

$$
\begin{aligned}
& 0>c_{\lambda, \mu}=I_{\lambda, \mu}\left(u_{1}, v_{1}\right)=\left(\frac{1}{2}-\frac{1}{2_{s}^{*}(\beta)}\right)\left\|\left(u_{1}, v_{1}\right)\right\|_{W}^{2} \\
& -\left(\frac{1}{q}-\frac{1}{2_{s}^{*}(\beta)}\right) Q_{\lambda, \mu}\left(u_{1}, v_{1}\right) \geq-\frac{2_{s}^{*}(\beta)-q}{q 2_{s}^{*}(\beta)} Q_{\lambda, \mu}\left(u_{1}, v_{1}\right) \\
& \geq-\frac{2_{s}^{*}(\beta)-q}{q 2_{s}^{*}(\beta)} \Lambda(s, \alpha)^{-\frac{q}{2}}\left\|\left(u_{1}, v_{1}\right)\right\|_{W}^{q} \\
& \times\left(\left(\lambda\|f\|_{L^{p}\left(\Omega,|x|^{-\alpha}\right)}\right)^{\frac{2}{2-q}}+\left(\mu\|g\|_{L^{p}\left(\Omega,|x|^{-\alpha}\right)}\right)^{\frac{2}{2-q}}\right)^{\frac{2-q}{2}} .
\end{aligned}
$$

This implies that $I_{\lambda, \mu}\left(u_{1}, v_{1}\right) \rightarrow 0$ as $\lambda \rightarrow 0^{+}$and $\mu \rightarrow 0^{+}$.

Next, we establish the existence of a local minimum for $I_{\lambda, \mu}$ on $\mathcal{N}_{\lambda, \mu}^{-}$.

Lemma 10. Under the assumptions of Theorem 2, there exist a nonnegative function $\left(u_{0}, v_{0}\right) \in W \backslash\{(0,0)\}$ and $\widehat{\Lambda}>0$ such that

$$
\sup _{t \geq 0} I_{\lambda, \mu}\left(t u_{0}, t v_{0}\right)<c_{\infty}
$$

for all $\lambda, \mu>0$ with $0<\left(\lambda\|f\|_{L^{p}\left(\Omega,|x|^{-\alpha}\right)}\right)^{\frac{2}{2-q}}+\left(\mu\|g\|_{L^{p}\left(\Omega,|x|^{-\alpha}\right)}\right)^{\frac{2}{2-q}}<\widehat{\Lambda}$, where $c_{\infty}$ is the constant given in (3.3). In particular, $c_{\lambda, \mu}^{-}<c_{\infty}$.

Proof. Now, we first consider the functional $J: W \rightarrow \mathbb{R}$ defined by

$$
J(u, v)=\frac{1}{2}\|(u, v)\|_{W}^{2}-\frac{1}{2_{s}^{*}(\beta)} \int_{\Omega} \frac{F(u, v)}{|x|^{\beta}} d x
$$

From Lemma 1 , there exists $\left(e_{1}, e_{2}\right) \in \mathbb{R}^{2}$ such that $e_{1}^{2}+e_{2}^{2}=1$ and $M_{F}=$ $F\left(e_{1}, e_{2}\right)^{\frac{2}{2_{s}^{*}(\beta)}}$. Set $u_{0}=e_{1} u_{\varepsilon}, v_{0}=e_{2} u_{\varepsilon}$, where $u_{\varepsilon}(x)=\varphi(x) U_{\varepsilon}(x), \varepsilon>0$, given by (2.10). Then, by $S_{F}(s, \beta)=M_{F}^{-1} \Lambda(s, \beta),(2.11),(2.12)$ and the fact

$$
\max _{t \geq 0}\left(\frac{t^{2}}{2} B_{1}-\frac{t^{2_{s}^{*}(\beta)}}{2_{s}^{*}(\beta)} B_{2}\right)=\frac{2_{s}^{*}(\beta)-2}{22_{s}^{*}(\beta)}\left(B_{1} B_{2}^{-\frac{2}{2_{s}^{*}(\beta)}}\right)^{\frac{2_{s}^{*}(\beta)}{2_{s}^{*}(\beta)-2}}, \quad B_{1} B_{2}>0
$$

we conclude that

$$
\begin{aligned}
& \sup _{t \geq 0} J\left(t u_{0}, t v_{0}\right) \leq \frac{2_{s}^{*}(\beta)-2}{22_{s}^{*}(\beta)}\left(\frac{\left(e_{1}^{2}+e_{2}^{2}\right)\left\|u_{\varepsilon}\right\|_{\gamma}^{2}}{\left(\int_{\Omega} \frac{F\left(e_{2} u_{\varepsilon}, e_{2} u_{\varepsilon}\right)}{|x|^{\beta}} d x\right)^{\frac{2}{2_{s}^{*}(\beta)}}}\right)^{\frac{2_{s}^{*}(\beta)}{2_{s}^{*}(\beta)-2}} \\
& =\frac{2_{s}^{*}(\beta)-2}{22_{s}^{*}(\beta)}\left(\frac{\left(e_{1}^{2}+e_{2}^{2}\right)\left\|u_{\varepsilon}\right\|_{\gamma}^{2}}{\left(M_{F}^{\frac{2_{s}^{*}(\beta)}{2}} \int_{\Omega} \frac{\left|u_{\varepsilon}\right|_{s}^{2 *}(\beta)}{|x|^{\beta}} d x\right)^{\frac{2_{s}^{*}(\beta)}{2_{s}^{*}(\beta)-2}}}\right)^{\frac{2}{2_{s}^{*}(\beta)}} \leq \frac{2_{s}^{*}(\beta)-2}{22_{s}^{*}(\beta)}\left(\frac{1}{M_{F}}\right)^{\frac{2_{s}^{*}(\beta)}{2_{s}^{*}(\beta)-2}}
\end{aligned}
$$




$$
\begin{aligned}
& \times\left[\frac{\Lambda(s, \beta)^{\frac{2_{s}^{*}(\beta)}{2_{s}^{*}(\beta)-2}}+O\left(\varepsilon^{2 b(\gamma)+2 s-N}\right)}{\left(\Lambda(s, \beta)^{\frac{2_{s}^{*}(\beta)}{2_{s}^{*}(\beta)-2}}+O\left(\varepsilon^{2_{s}^{*}(\beta) b(\gamma)+\beta-N}\right)\right)^{\frac{2}{2_{s}^{*}(\beta)}}}\right]^{\frac{2_{s}^{*}(\beta)}{2_{s}^{*}(\beta)-2}} \\
\leq & \frac{2 s-\beta}{2(N-\beta)} S_{F}(s, \beta)^{\frac{2_{s}^{*}(\beta)}{2_{s}^{*}(\beta)-2}}+O\left(\varepsilon^{2 b(\gamma)+2 s-N}\right) .
\end{aligned}
$$

Let $C_{0}$ be a positive constant given in Lemma 2, we can choose $\Lambda_{2}>0$ small enough such that $c_{\infty}>0$ for all $\lambda, \mu>0$ with $0<\left(\lambda\|f\|_{L^{p}\left(\Omega,|x|^{-\alpha}\right)}\right)^{\frac{2}{2-q}}+$ $\left(\mu\|g\|_{L^{p}\left(\Omega,|x|^{-\alpha}\right)}\right)^{\frac{2}{2-q}}<\Lambda_{2}$. Using the definition of $I_{\lambda, \mu}(u, v)$, we have

$$
I_{\lambda, \mu}\left(t u_{0}, t v_{0}\right) \leq \frac{t^{2}}{2}\left\|\left(u_{0}, v_{0}\right)\right\|_{W}^{2} \leq C t^{2}, \quad \forall t \geq 0, \lambda, \mu>0,
$$

which implies that there exists $t_{0} \in(0,1)$ such that

$$
\sup _{t \in\left[0, t_{0}\right]} I_{\lambda, \mu}\left(t u_{0}, t v_{0}\right)<c_{\infty}
$$

for all $\lambda, \mu>0$ with $0<\left(\lambda\|f\|_{L^{p}\left(\Omega,|x|^{-\alpha}\right)}\right)^{\frac{2}{2-q}}+\left(\mu\|g\|_{L^{p}\left(\Omega,|x|^{-\alpha}\right)}\right)^{\frac{2}{2-q}}<\Lambda_{2}$.

Next, we prove that $\sup I_{\lambda, \mu}\left(t u_{0}, t v_{0}\right)<c_{\infty}$. Since $f(x), g(x) \geq a_{0}$ for all $x \in B_{r_{0}}(0) \subset \Omega$, we have

$$
\begin{aligned}
Q_{\lambda, \mu}\left(u_{0}, v_{0}\right) & =\lambda e_{1}^{q} \int_{\Omega} \frac{f(x)\left|u_{\varepsilon}\right|^{q}}{|x|^{\alpha}} d x+\mu e_{2}^{q} \int_{\Omega} \frac{g(x)\left|u_{\varepsilon}\right|^{q}}{|x|^{\alpha}} d x \\
& \geq a_{0} M(\lambda+\mu) \int_{B_{\rho}(0)} \frac{\left|u_{\varepsilon}\right|^{q}}{|x|^{\alpha}} d x
\end{aligned}
$$

where $M=\min \left\{e_{1}^{q}, e_{2}^{q}\right\}$. Combining (5.6), (5.8) and (2.13), for all $t \geq t_{0}$, we get

$$
\begin{aligned}
& \sup _{t \geq t_{0}} I_{\lambda, \mu}\left(t u_{0}, t v_{0}\right)=\sup _{t \geq t_{0}}\left(J\left(t u_{0}, t v_{0}\right)-\frac{t^{q}}{q} Q_{\lambda, \mu}\left(u_{0}, v_{0}\right)\right) \\
& \leq \frac{2 s-\beta}{2(N-\beta)} S_{F}(s, \beta)^{\frac{2_{s}^{*}(\beta)}{2_{s}^{*}(\beta)-2}}+O\left(\varepsilon^{2 b(\gamma)+2 s-N}\right)-\frac{t_{0}^{q}}{q} a_{0} M(\lambda+\mu) \int_{B_{\rho}(0)} \frac{\left|u_{\varepsilon}\right|^{q}}{|x|^{\alpha}} d x \\
& \leq \frac{2 s-\beta}{2(N-\beta)} S_{F}(s, \beta)^{\frac{2_{s}^{*}(\beta)}{2 s_{s}^{*}(\beta)-2}}+O\left(\varepsilon^{2 b(\gamma)+2 s-N}\right) \\
& -(\lambda+\mu)\left\{\begin{array}{lll}
C \varepsilon^{N-\alpha-\frac{q(N-2 s)}{2}}, & \text { if } \quad q>(N-\alpha) / b(\gamma), \\
C \varepsilon^{\left.N-\alpha-\frac{q(N-2 s)}{2}\right)}|\ln \varepsilon|, & \text { if } \quad q=(N-\alpha) / b(\gamma), \\
C \varepsilon^{q\left(b(\gamma)-\frac{N-2 s}{2}\right)}, & \text { if } \quad q<(N-\alpha) / b(\gamma) .
\end{array}\right.
\end{aligned}
$$

(i) If $1 \leq q<\frac{N-\alpha}{b(\gamma)}$. Since $b(\gamma)>\frac{N-2 s}{2}$, we get $2 b(\gamma)+2 s-N>q\left(b(\gamma)-\frac{N-2 s}{2}\right)$. Then, for $\varepsilon$ small enough, we can choose $\Lambda_{3}>0$ such that

$$
\begin{aligned}
O\left(\varepsilon^{2 b(\gamma)+2 s-N}\right) & -C(\lambda+\mu) \varepsilon^{q\left(b(\gamma)-\frac{N-2 s}{2}\right)} \\
& <-C_{0}\left(\left(\lambda\|f\|_{L^{p}\left(\Omega,|x|^{-\alpha}\right)}\right)^{\frac{2}{2-q}}+\left(\mu\|g\|_{L^{p}\left(\Omega,|x|^{-\alpha}\right)}\right)^{\frac{2}{2-q}}\right)
\end{aligned}
$$


for all $\lambda, \mu>0$ with $0<\left(\lambda\|f\|_{L^{p}\left(\Omega,|x|^{-\alpha}\right)}\right)^{\frac{2}{2-q}}+\left(\mu\|g\|_{L^{p}\left(\Omega,|x|^{-\alpha}\right)}\right)^{\frac{2}{2-q}}<\Lambda_{3}$. Set $\Lambda_{4}=\min \left\{\Lambda_{2}, \Lambda_{3}\right\}$, then for all $\lambda, \mu>0$ with $0<\left(\lambda\|f\|_{L^{p}\left(\Omega,|x|^{-\alpha}\right)}\right)^{\frac{2}{2-q}}+$ $\left(\mu\|g\|_{L^{p}\left(\Omega,|x|^{-\alpha}\right)}\right)^{\frac{2}{2-q}}<\Lambda_{4},(5.7),(5.9)$ and (5.10) show that

$$
\sup _{t \geq 0} I_{\lambda, \mu}\left(t u_{0}, t v_{0}\right)<c_{\infty}
$$

(ii) If $\frac{N-\alpha}{b(\gamma)} \leq q<2$. From $b(\gamma)>\frac{N-2 s}{2}$ and $\frac{N-\alpha}{b(\gamma)} \leq q$, we can obtain $2 b(\gamma)+2 s-N>N-\alpha-\frac{q(N-2 s)}{2}$. Then, for $\varepsilon$ small enough, there exists a $\Lambda_{5}>0$ such that

$$
\begin{aligned}
O\left(\varepsilon^{2 b(\gamma)+2 s-N}\right) & -C(\lambda+\mu) \varepsilon^{N-\alpha-\frac{q(N-2 s)}{2}} \\
& <-C_{0}\left(\left(\lambda\|f\|_{L^{p}\left(\Omega,|x|^{-\alpha}\right)}\right)^{\frac{2}{2-q}}+\left(\mu\|g\|_{L^{p}\left(\Omega,|x|^{-\alpha}\right)}\right)^{\frac{2}{2-q}}\right)
\end{aligned}
$$

for all $\lambda, \mu>0$ with $0<\left(\lambda\|f\|_{L^{p}\left(\Omega,|x|^{-\alpha}\right)}\right)^{\frac{2}{2-q}}+\left(\mu\|g\|_{L^{p}\left(\Omega,|x|^{-\alpha}\right)}\right)^{\frac{2}{2-q}}<\Lambda_{5}$. Similarly, let $\Lambda_{6}=\min \left\{\Lambda_{2}, \Lambda_{5}\right\}$, by (5.7), (5.9) and (5.11), one can get

$$
\sup _{t \geq 0} I_{\lambda, \mu}\left(t u_{0}, t v_{0}\right)<c_{\infty}
$$

for all $\lambda, \mu>0$ with $0<\left(\lambda\|f\|_{L^{p}\left(\Omega,|x|^{-\alpha}\right)}\right)^{\frac{2}{2-q}}+\left(\mu\|g\|_{L^{p}\left(\Omega,|x|^{-\alpha}\right)}\right)^{\frac{2}{2-q}}<\Lambda_{6}$.

Set $\widehat{\Lambda}=\min \left\{\Lambda_{4}, \Lambda_{6}\right\}$, from cases (i) and (ii), (5.5) holds by taking $\left(u_{0}, v_{0}\right)=$ $\left(e_{1} u_{\varepsilon}, e_{2} u_{\varepsilon}\right)$ and for all $0<\left(\lambda\|f\|_{L^{p}\left(\Omega,|x|^{-\alpha}\right)}\right)^{\frac{2}{2-q}}+\left(\mu\|g\|_{L^{p}\left(\Omega,|x|^{-\alpha}\right)}\right)^{\frac{2}{2-q}}<\widehat{\Lambda}$.

Recalling that $\left(u_{0}, v_{0}\right)=\left(e_{1} u_{\varepsilon}, e_{2} u_{\varepsilon}\right)$, it is easy to see that

$$
\int_{\Omega} \frac{F\left(u_{0}, v_{0}\right)}{|x|^{\beta}} d x>0 \text { and } Q_{\lambda, \mu}\left(u_{0}, v_{0}\right)>0 \text {. }
$$

Then, from (5.12) and Lemma 8, we get that there exists $t^{-}>0$ such that $\left(t^{-} u_{0}, t^{-} v_{0}\right) \in \mathcal{N}_{\lambda, \mu}^{-}$. Thus, it follows from the definition of $c_{\lambda, \mu}^{-}$and (5.5) that $\left.c_{\lambda, \mu}^{-} \leq I_{\lambda, \mu}\left(t^{-} u_{0}, t^{-} v_{0}\right) \leq \sup _{t \geq 0} I_{\lambda, \mu}\left(t u_{0}, t v_{0}\right)\right)<c_{\infty}$ for all $\lambda, \mu>0$ with $0<$ $\left(\lambda\|f\|_{L^{p}\left(\Omega,|x|^{-\alpha}\right)}\right)^{\frac{2}{2-q}}+\left(\mu\|g\|_{L^{p}\left(\Omega,|x|^{-\alpha}\right)}\right)^{\frac{2}{2-q}}<\widehat{\Lambda}$. The proof is thus complete.

Now, we establish the existence of a local minimum of $I_{\lambda, \mu}$ on $\mathcal{N}_{\lambda, \mu}^{-}$.

Theorem 4. Set $\Lambda^{*}=\min \left\{\Lambda_{1}, \widehat{\Lambda}\right\}$, under the assumptions of Theorem 2, the problem (1.1) has a positive solution $\left(u_{2}, v_{2}\right) \in \mathcal{N}_{\lambda, \mu}^{-}$and $I_{\lambda, \mu}\left(u_{2}, v_{2}\right)=c_{\lambda, \mu}^{-}$for all $\lambda, \mu>0$ with $0<\left(\lambda\|f\|_{L^{p}\left(\Omega,|x|^{-\alpha}\right)}\right)^{\frac{2}{2-q}}+\left(\mu\|g\|_{L^{p}\left(\Omega,|x|^{-\alpha}\right)}\right)^{\frac{2}{2-q}}<\Lambda^{*}$.

Proof. $\quad$ Set $\Lambda^{*}=\min \left\{\Lambda_{1}, \widehat{\Lambda}\right\}$. By Proposition 9 (ii), Lemmas 3 and 10, for all $\lambda, \mu>0$ with $0<\left(\lambda\|f\|_{L^{p}\left(\Omega,|x|^{-\alpha}\right)}\right)^{\frac{2}{2-q}}+\left(\mu\|g\|_{L^{p}\left(\Omega,|x|^{-\alpha}\right)}\right)^{\frac{2}{2-q}}<\Lambda^{*}, I_{\lambda, \mu}$ satisfies the $(P S)_{c_{\lambda, \mu}^{-}}$condition for all $c_{\lambda, \mu}^{-} \in\left(0, c_{\infty}\right)$. Since $I_{\lambda, \mu}$ is coercive on $\mathcal{N}_{\lambda, \mu}^{-}$, we get that the $(P S)_{c_{\lambda, \mu}^{-}}$-sequence $\left\{\left(u_{n}, v_{n}\right)\right\}$ is bounded. Therefore, there exist a subsequence still denoted by $\left\{\left(u_{n}, v_{n}\right)\right\}$ and $\left(u_{2}, v_{2}\right) \in W \backslash\{(0,0)\}$ 
such that $\left(u_{n}, v_{n}\right) \rightarrow\left(u_{2}, v_{2}\right)$ weakly in $W$. Arguing as in the proof of Theorem 3 , we obtain $\left(u_{n}, v_{n}\right) \rightarrow\left(u_{2}, v_{2}\right)$ strongly in $W$ and $\left(u_{2}, v_{2}\right)$ is a positive solution of (1.1) for all $\lambda, \mu>0$ with $0<\left(\lambda\|f\|_{L^{p}\left(\Omega,|x|^{-\alpha}\right)}\right)^{\frac{2}{2-q}}+\left(\mu\|g\|_{L^{p}\left(\Omega,|x|^{-\alpha}\right)}\right)^{\frac{2}{2-q}}<$ $\Lambda^{*}$.

Finally, we prove that $\left(u_{2}, v_{2}\right) \in \mathcal{N}_{\lambda, \mu}^{-}$. Arguing by contradiction, we assume $\left(u_{2}, v_{2}\right) \in \mathcal{N}_{\lambda, \mu}^{+}$. Since $\mathcal{N}_{\lambda, \mu}^{-}$is closed in $W$, we have $\left\|\left(u_{2}, v_{2}\right)\right\|_{W}<$ $\liminf _{n \rightarrow \infty}\left\|\left(u_{n}, v_{n}\right)\right\|_{W}$. Moreover, by Lemma 8 , there exists a unique $t_{2}^{-}$such that $\left(t_{2}^{-} u_{2}, t_{2}^{-} v_{2}\right) \in \mathcal{N}_{\lambda, \mu}^{-}$. This and $\left(u_{n}, v_{n}\right) \in \mathcal{N}_{\lambda, \mu}^{-}$deduce that

$$
c_{\lambda, \mu}^{-} \leq I_{\lambda, \mu}\left(t_{2}^{-} u_{2}, t_{2}^{-} v_{2}\right)<\lim _{n \rightarrow \infty} I_{\lambda, \mu}\left(t_{2}^{-} u_{n}, t_{2}^{-} v_{n}\right) \leq \lim _{n \rightarrow \infty} I_{\lambda, \mu}\left(u_{n}, v_{n}\right)=c_{\lambda, \mu}^{-} .
$$

So, $\left(u_{2}, v_{2}\right) \in \mathcal{N}_{\lambda, \mu}^{-}$. This completes the proof of Theorem 4 .

Proof of Theorem 2 (ii). By Theorem 3, the system (1.1) has a positive solution $\left(u_{1}, v_{1}\right) \in \mathcal{N}_{\lambda, \mu}^{+}$for all $\lambda, \mu>0$ with $0<\left(\lambda\|f\|_{L^{p}\left(\Omega,|x|^{-\alpha}\right)}\right)^{\frac{2}{2-q}}+$ $\left(\mu\|g\|_{L^{p}\left(\Omega,|x|^{-\alpha}\right)}\right)^{\frac{2}{2-q}}<\Lambda_{0}$. On the other hand, from Theorem 4, we can get the second positive solution $\left(u_{2}, v_{2}\right) \in \mathcal{N}_{\lambda, \mu}^{-}$for all $\lambda, \mu>0$ with $0<$ $\left(\lambda\|f\|_{L^{p}\left(\Omega,|x|^{-\alpha}\right)}\right)^{\frac{2}{2-q}}+\left(\mu\|g\|_{L^{p}\left(\Omega,|x|^{-\alpha}\right)}\right)^{\frac{2}{2-q}}<\Lambda^{*}$. Since $\mathcal{N}_{\lambda, \mu}^{+} \cap \mathcal{N}_{\lambda, \mu}^{-}=\emptyset$ and $\Lambda^{*}<\Lambda_{0}$, we get $\left(u_{1}, v_{1}\right),\left(u_{2}, v_{2}\right)$ are distinct positive solutions of (1.1) for all $\lambda, \mu>0$ with $0<\left(\lambda\|f\|_{L^{p}\left(\Omega,|x|^{-\alpha}\right)}\right)^{\frac{2}{2-q}}+\left(\mu\|g\|_{L^{p}\left(\Omega,|x|^{-\alpha}\right)}\right)^{\frac{2}{2-q}}<\Lambda^{*}$. This completes the proof of Theorem 2 (ii).

\section{References}

[1] G.M. Bisci, G. Molica, V.D. Radulescu and R. Servadei. Variational methods for nonlocal fractional problems. Vol. 162. Cambridge University Press, 2016.

[2] X. Cabre and Y. Sire. Nonlinear equations for fractional Laplacians, I: regularity, maximum principles, and Hamiltonian estimates. Ann Inst H Poincaré Anal Non Linéaire, 31:23-53, 2014. https://doi.org/10.1016/j.anihpc.2013.02.001.

[3] L. Cai, J. Liang and J. Zhang. Properties of solutions for a coupled fractional nonlinear system. J. Nonlinear and Convex Anal., 19:323-344, 2018.

[4] D.C. de M. Filho and M.A.S. Souto. Systems of $p$-Laplacian equations involving homogenous nonlinearities with critical Sobolev exponent degrees. Comm. Part. Diff. Equ., 24:1537-1553, 1999. https://doi.org/10.1080/03605309908821473.

[5] P. Drabek and S.I. Pohozaev. Positive solutions for the p-Laplacian: application of the fibering method. Proc. Roy. Soc. Edinburh Sect. A, 127:703-727, 1997. https://doi.org/10.1017/S0308210500023787.

[6] N. Ghoussoub, F. Robert, S. Shakerian and M. Zhao. Mass and asymptotics associated to fractional Hardy-Schrödinger operators in critical regimes. Comm. Part. Diff. Equ., 43(6):859-892, 2018. https://doi.org/10.1080/03605302.2018.1476528.

[7] N. Ghoussoub and S. Shakerian. Borderline variational problems involving fractional Laplacians and critical singularities. Adv. Nonl. Studies, 15:527-555, 2015. https://doi.org/10.1515/ans-2015-0302. 
[8] J. Giacomoni, T. Mukherjee and K. Sreenadh. Doubly nonlocal system with Hardy-Littlewood-Sobolev critical nonlinearity. J. Math. Anal. Appl., 467:638672, 2018. https://doi.org/10.1016/j.jmaa.2018.07.035.

[9] S. Goyal and K. Sreenadh. Existence and multiplicity of solutions for $p$-fractional Laplace equation with sign-changing nonlinearities. Adv. Nonl. Anal., 4(1):3758, 2015. https://doi.org/10.1515/anona-2014-0017.

[10] S. Goyal and K. Sreenadh. Nehari manifold for non-local elliptic operator with concave-convex nonlinearities and sign-changing weight functions. Proceedings of Indian Academy of Sci., 125(6):545-558, 2015. https://doi.org/10.1007/s12044015-0244-5.

[11] T.-S. Hsu. Multiple positive solutions for a quasilinear elliptic problem involving critical Sobolev-Hardy exponents and concave-convex nonlinearities. Nonlinear Anal., 74:3934-3944, 2011. https://doi.org/10.1016/j.na.2011.02.036.

[12] T.-S. Hsu and H.-L. Lin. Multiple positive solutions for singular elliptic equations with weighted Hardy terms and critical Sobolev-Hardy exponents. Proc. Roy. Soc. Edinburgh Sect. A., 140:617-633, 2010. https://doi.org/10.1017/S0308210509000729.

[13] T.-S. Hsu and H.-L. Lin. Multiplicity of positive solutions for semilinear elliptic systems with critical Sobolev-Hardy and Concave exponents. Acta. Math. Sci., 31(B):791-804, 2011. https://doi.org/10.1016/S0252-9602(11)60276-2.

[14] T.-S. Hsu, H.-L. Lin and C.-C Hu. Multiple positive solutions of quasilinear elliptic equations in $\mathbb{R}^{N}$. J. Math. Anal. Appl., 388:500-512, 2012.

[15] T. Mukherjee and K. Sreenadh. On doubly nonlocal $p$-fractional coupled elliptic system. Topological Meth. Nonl. Anal., 51(2):609-636, 2018. https://doi.org/10.12775/TMNA.2018.018.

[16] E. Di Nezza, G. Palatucci and E. Valdinoci. Hitchhikers guide to the fractional Sobolev spaces. Bull Sci. Math., 136:521-573, 2012. https://doi.org/10.1016/j.bulsci.2011.12.004.

[17] X. Ros-Oton and J. Serra. The Pohozaev identity for the fractional Laplacian. Arch. Rational Mech. Anal., 213:587-628, 2014. https://doi.org/10.1007/s00205-014-0740-2.

[18] S. Shakerian. Multiple positive solutions for nonlocal elliptic problems involving the Hardy potential and concave-convex nonlinearities. Cornell University, 2017. Available from Internet: https://arxiv.org/abs/1708.01369.

[19] L. Silvestre. Regularity of the obstacle problem for a fractional power of the Laplace operator. Com. Pure Appl. Math., 60:67-112, 2007. https://doi.org/10.1002/cpa.20153.

[20] J. Zhang and T.-S. Hsu. Nonlocal elliptic systems involving critical SobolevHardy exponents and concave-convex nonlinearities. Taiwanese J. Math., 2019. https://doi.org/10.11650/tjm/190109.

[21] J. Zhang and X. Liu. Three solutions for a fractional elliptic problems with critical and supercritical growth. Acta Math. Sci., 36B(6):1-13, 2016. https://doi.org/10.1016/S0252-9602(16)30108-4.

[22] J. Zhang, X. Liu and H. Jiao. Multiplicity of positive solutions for a fractional Laplacian equations involving critical nonlinearity. Topological Meth. Nonl. Anal., 53(1):151-182, 2019. https://doi.org/10.12775/TMNA.2018.043. 